ARTICLE

DOI: $10.1038 / s 41467-017-00619-z$

\title{
Mapping surface-modified titania nanoparticles with implications for activity and facet control
}

Yung-Kang Peng ${ }^{1}$, Yichen $\mathrm{Hu}^{2}$, Hung-Lung Chou ${ }^{3}$, Yingyi Fu², Ivo F. Teixeira', Li Zhang ${ }^{2}$, Heyong He ${ }^{2} \&$ Shik Chi Edman Tsang (1) ${ }^{1}$

The use of surface-directing species and surface additives to alter nanoparticle morphology and physicochemical properties of particular exposed facets has recently been attracting significant attention. However, challenges in their chemical analysis, sometimes at trace levels, and understanding their roles to elucidate surface structure-activity relationships in optical (solar cells) or (photo)catalytic performance and their removal are significant issues that remain to be solved. Here, we show a detailed analysis of $\mathrm{TiO}_{2}$ facets promoted with surface species $\left(\mathrm{OH}, \mathrm{O}, \mathrm{SO}_{4}, \mathrm{~F}\right)$ with and without post-treatments by ${ }^{31} \mathrm{P}$ adsorbate nuclear magnetic resonance, supported by a range of other characterization tools. We demonstrate that quantitative evaluations of the electronic and structural effects imposed by these surface additives and their removal mechanisms can be obtained, which may lead to the rational control of active $\mathrm{TiO}_{2}$ (001) and (101) facets for a range of applications.

\footnotetext{
${ }^{1}$ The Wolfson Catalysis Centre, Department of Chemistry, University of Oxford, Oxford OX1 3QR, UK. ${ }^{2}$ Department of Chemistry and Shanghai Key Laboratory of Molecular Catalysis and Innovative Materials, Fudan University, Shanghai 200433, People's Republic of China. ${ }^{3}$ Graduate Institute of Applied Science and Technology, National Taiwan University of Science and Technology, Taipei 10617, Taiwan. Correspondence and requests for materials should be addressed to S.C.E.T. (email: edman.tsang@chem.ox.ac.uk)
} 
S urface features and distributions on metal oxides such as oxygen vacancies $\left(\mathrm{V}_{\mathrm{o}}\right)$, cations, anions and hydroxyl groups have been shown to play a decisive role in various important applications such as optics, electronic devices and heterogeneous catalysis ${ }^{1-5}$. In particular, for nano-sized particles, the domination of these surface features has been demonstrated to lead to the particles exhibiting different physical/chemical properties compared with the bulk. Given that each facet possesses a distinctive intrinsic energy, it is understandable that both the concentration and chemical state of the surface features differ from facet to facet. Taking $\mathrm{ZnO}$ nanocrystallite as an example, the surface $V_{o}$ were shown to be present on the oxygen-terminated (002) facet $^{6}$, and were recently quantified by Peng et al. ${ }^{7}$ Also, the chemical properties of surface zinc ions have been shown to be different on (100) and (002) facets, indicating the Lewis acidity of $\mathrm{ZnO} \mathrm{NP}$ can be modulated by the morphology control ${ }^{7}$. These considerations have provided additional variables in tailoring the morphology of nanoparticles (NPs) with preferentially exposed facets during the past decades. Unfortunately, facets with high reactivity are unstable and tend to diminish rapidly to minimize surface energy during the crystal growth $^{8}$. Accordingly, a structure-directing species (SDS) or surfactant is usually employed in the shape control of NPs, which renders them in metastable and high-energy forms. The SDS can subtly modify the chemical state of the surface features (especially metal cations) and provide a kinetic growth control for the formation of shaped $\mathrm{NPs}^{1}$. As the growth rate of a crystal facet depends on the surface energy, different morphologies of NP could thus be prepared by using various $\operatorname{SDS}^{8}$, 9 .

The tailoring of anatase $\mathrm{TiO}_{2}$ crystals with the termination of specific facets has received great interest for many years. However, most synthetic $\mathrm{TiO}_{2}$ crystals are covered with thermodynamically more stable (101) facets rather than the reactive $(001)$ facets ${ }^{10,11}$. The breakthrough in controlling the synthesis of crystals with a high percentage of reactive highenergy (001) sites was not made until $2008^{12}$. According to that literature, the relative stability of these two facets can be reversed using a small quantity of fluorine on the (001) surface during the particle synthesis. Since then, the controlled synthesis of $\mathrm{TiO}_{2}$ nanocrystals with preferentially exposed (001) facets has been shown to display promising properties in photocatalysis ${ }^{13-24}$, methanol conversion ${ }^{25}$, solar cells ${ }^{26-29}$ and lithium batteries ${ }^{30,31}$, etc. Generally the removal of SDS, with fluorine in this case, after the controlled nanoparticle synthesis is required to avoid interference with the desired surface properties. It can be removed by a simple post-treatment step such as calcination at $600{ }^{\circ} \mathrm{C}^{12}, 14-16,19,21,23,26,27,30$ or ion exchange with aqueous $\mathrm{NaOH}^{13}, 15,17-19,24$ before the application to obtain a so-called 'clean surface'. However, by adopting different removal methods, diverse results have been obtained in the literature in various applications (e.g., photocatalysis cases summarized in Table 1), which have led to different interpretations and frequent disagreements among researchers ${ }^{13-31}$. For example, the $\mathrm{NaOH}$ treated (001) facet (i.e., Na-(001)) showed higher activity in the photocatalytic degradation of methyl orange dye than the F-capped (001) facet (i.e., F- $(001))^{13}$, while a reverse result was obtained for the same reaction by another research group ${ }^{15}$ (Table 1). Also, both $\mathrm{NaOH}$ and calcination treatments were reported to increase the facet activity of (101) in photocatalytic hydrogen evolution ${ }^{16,17}$, while the $\mathrm{NaOH}$ treatment was concluded to lower the facet activity of (001) in this reaction by others ${ }^{17,18}$. These different observations can arise from the surface reconstruction of the unstable (001) surface upon its removal during calcination ${ }^{32}, 33$ or traces of residual SDS that remain on the surface affecting the chemical state of exposed titanium (vide infra). So far, no clear rationalization or guidance for the selection of appropriate post-treatment methods has been achieved. In addition, the SDS modification on the $\mathrm{TiO}_{2}$ surface has been demonstrated to efficiently modulate the surface $\mathrm{Ti}$ chemical state. For example, the post-modification of $\mathrm{PO}_{4}{ }^{3-} / \mathrm{SO}_{4}{ }^{2-}$ on the $\mathrm{TiO}_{2}$ surface can provide extra Brønsted acid sites and also increase Lewis acidity for enhanced sugar conversion $^{34,35} /$ photocatalysis $^{36}, 37$. The chelating of surface $\mathrm{COOH}$-containing dye molecule ${ }^{38}$ and electrolyte additive ${ }^{39}$, 4 - $t$-butylpyridine, have been found to remarkably improve the solar cell performance due to the modification of the $\mathrm{TiO}_{2}$ conduction band (unfilled d-band of $\mathrm{Ti}^{4+}$ ) for efficient electron transfer in dye-sensitized solar cells.

Given the importance of the facet-dependent properties of $\mathrm{TiO}_{2}$ nanoparticles and their chemical modifications for a wide range of applications, to the best of our knowledge there is still no detailed work on the mapping of surface features of functionalized $\mathrm{TiO}_{2}$ with different morphologies. Herein, using anatase $\mathrm{TiO}_{2}$ samples of different morphologies, we successfully monitor the subtle changes of the $\mathrm{Ti}$ chemical state induced by a small quantity of surface adsorbates (i.e., fluorine, sulfate, $\mathrm{OH}$ ) on various facets using ${ }^{31} \mathrm{P}$ magic angle spinning (MAS) nuclear magnetic resonance (NMR) in combination with

Table 1 Facet-dependent photocatalytic reaction of anatase $\mathrm{TiO}_{2} \mathrm{NPs}$ prepared using fluorine as SDS

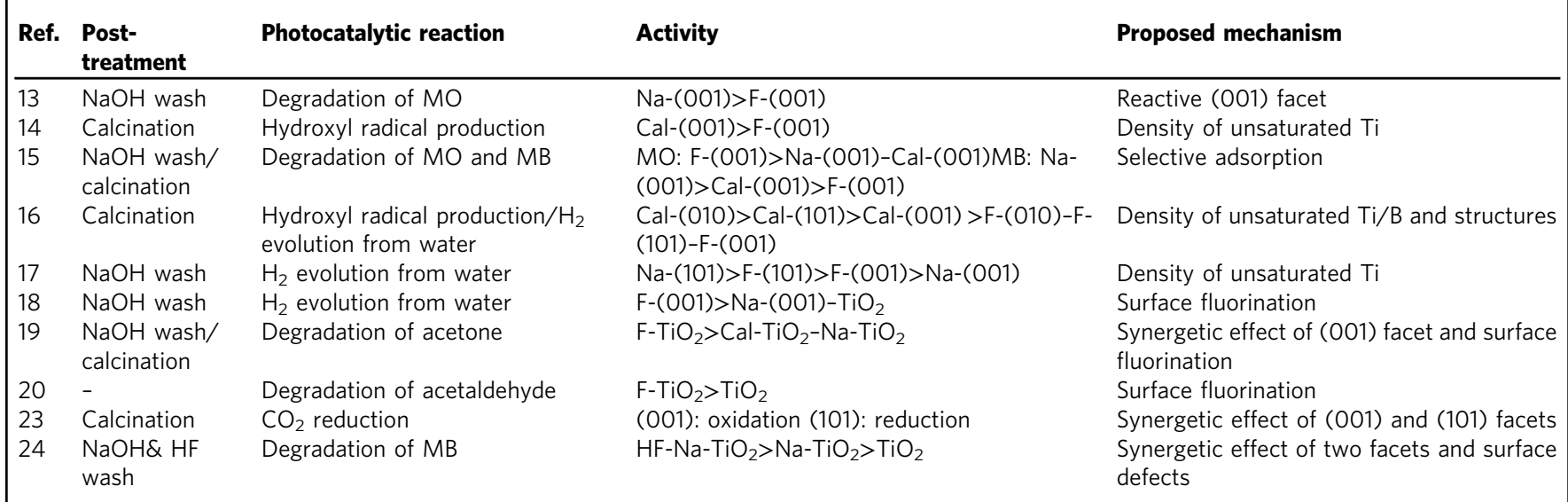

Facet-controlled fluorine-capped anatase $\mathrm{TiO}_{2} \mathrm{NPs}$ treated with various post-treatments ( $\mathrm{Na} \mathrm{NaOH}$ wash, $\mathrm{Cal}$ calcination) and their corresponding activities/mechanisms. $\mathrm{F}$-(001) represents asprepared F-capped $\mathrm{TiO}_{2} \mathrm{NP}$ preferentially exposes (001) facet; $\mathrm{Na} / \mathrm{Cal}-(001)$ represents $\mathrm{F}-(001)$ post-treated with $\mathrm{NaOH}$ wash/calcination for the removal of surface fluorine 

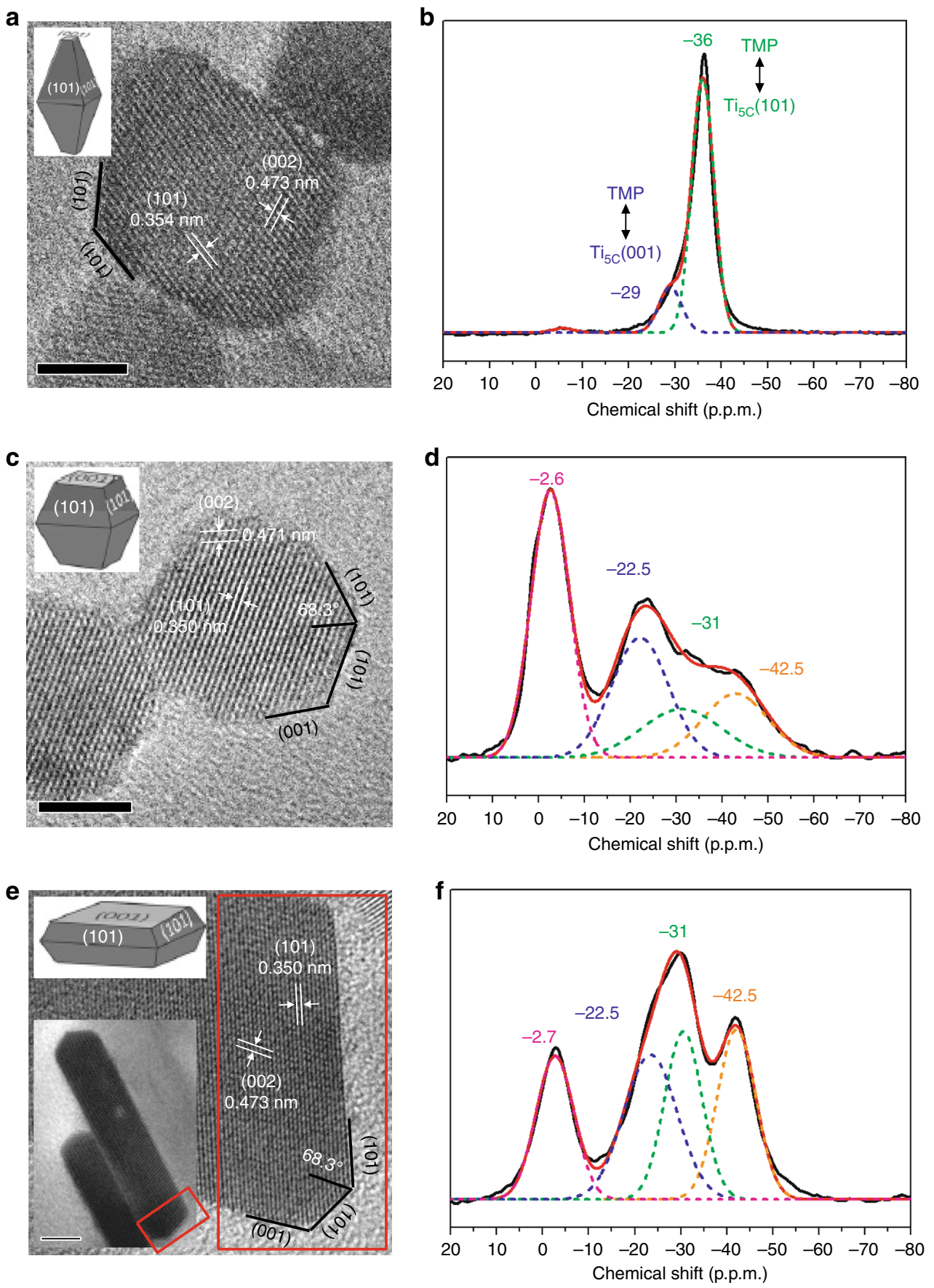

Fig. 1 TEM images of as-prepared $\mathrm{TiO}_{2}$ samples and corresponding ${ }^{31} \mathrm{P}$ MAS NMR spectra of TMP-adsorbed samples. TEM images of as-prepared a powder (PD), c F-(101), e F-(001) and $\mathbf{b}, \mathbf{d}, \mathbf{f}$ their corresponding spectra of TMP-adsorbed samples. Green represents TMP of surface 5-coordinate Ti ${ }^{4+}$ on (101) facet (i.e., $\mathrm{Ti}_{5 \mathrm{C}}(101)$ ), blue represents $\mathrm{TMP}$ of 5 -coordinate $\mathrm{Ti}^{4+}$ on $(001)$ facet (i.e., $\mathrm{Ti}_{5 \mathrm{C}}(001)$ ), orange represents $\mathrm{TMP}^{\mathrm{N}}$ of surface $\mathrm{Ti}^{4+}$ containing $\mathrm{OH}$ and $\mathrm{F}$ and pink represents TMP on Brønsted acid site ( $\mathrm{TiO}(\mathrm{H})-\mathrm{Ti})$. The $\mathrm{F}-(001)$ sample clearly show a higher coverage of (001) facet reflected by the largest corresponding ${ }^{31} \mathrm{P}$ chemical shift of blue peak (cf., green peak); while F-(101) with higher coverage of (101) facet and PD with the highest coverage of (101) facet both show a dominant green peak. See NMR spectrum deconvolution section in Experimental section for details. The scale bar in all images is $5 \mathrm{~nm}$

trimethylphosphine (TMP) as a surface probe. This is based on the fact that the nucleophilic probe TMP molecule can form a stable adduct with the exposed Ti cation (Lewis acid (LA)) of the $\mathrm{TiO}_{2}$ surface, and the formation of a surface TMP-Ti complex can be realized via coordination of the $\mathrm{P}$ atom to the surface Ti cation centre. According to ${ }^{31} \mathrm{P}$ chemical shift $\left(\delta^{31} \mathrm{P}\right)$ of the corresponding surface TMP-Ti complexes, it is demonstrated the surface Ti cations on various facets with different Lewis acidities, surface energies and steric arrangements can be carefully mapped, differentiated and quantitatively analysed. It is shown that some typical post-treatments (i.e., calcination or $\mathrm{NaOH}$ wash) to remove the fluorine (as SDS) can also lead to different surface Ti chemical states. According to our catalytic testing, extra caution must be exercised for even trivial surface treatments as a huge difference in activity can be obtained. This technique (i.e., using TMP as a surface probe) is thus demonstrated as a powerful tool in combination with conventional surface techniques adopted for $\mathrm{TiO}_{2} \mathrm{NPs}$ such as X-ray photoelectron spectroscopy (XPS), 


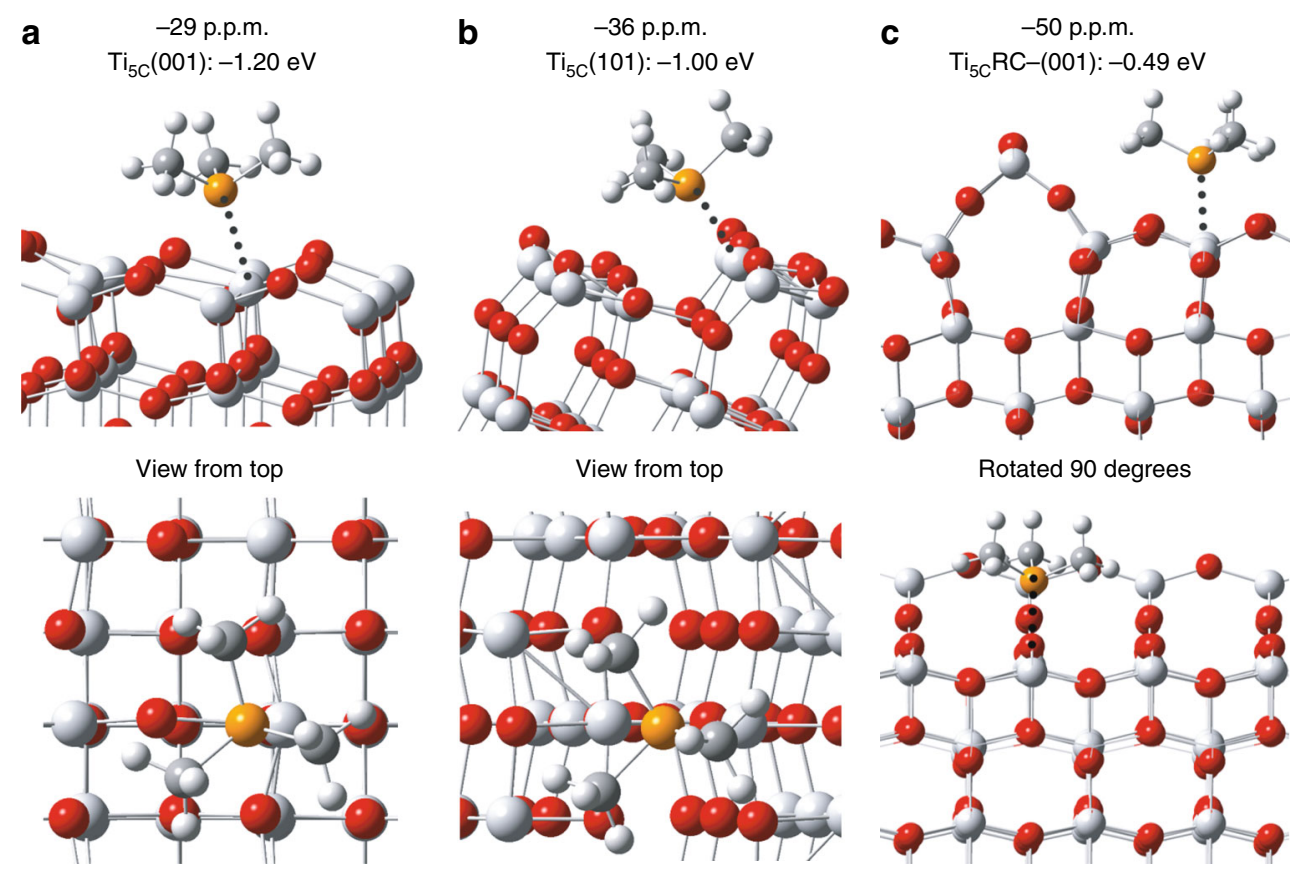

Fig. 2 Calculated results of molecular interaction. Schematic illustrations of molecular interaction and DFT calculated adsorption energy ( $\left.E_{a d}\right)$ between TMP and various $\mathrm{TiO}_{2}$ facets. a Ti ${ }_{5 \mathrm{C}}(001), \mathbf{b} \mathrm{Ti}_{5 \mathrm{C}}(101)$ and $\mathbf{c} \mathrm{Ti}_{5 \mathrm{C}} \mathrm{RC}-(001)$. RC-(001) represents reconstructed (1×4) (001) facet (Ti: light grey; O: red; $\mathrm{P}$ : orange; C: grey; $\mathrm{H}$ : white)

electron paramagnetic resonance (EPR) and Raman spectroscopy to offer comprehensive information of surface features on various functionalized facets to guide surface treatments/modifications of $\mathrm{TiO}_{2} \mathrm{NP}$ for various applications.

\section{Results}

$\mathrm{TiO}_{2}$ nanocrystals and their surface chemical states. Samples prepared with 0,2 and $6 \mathrm{~mL}$ of $50 \mathrm{wt} \%$ concentration of hydrogen fluoride (HF) reveal different morphologies and are labelled as powder (PD) (Fig. 1a), F-(101) (Fig. 1c) and F-(001) (Fig. 1e). All samples exhibited lattice fringes with d-spacings around 0.47 and $0.35 \mathrm{~nm}$, in accordance with the [101] and [002] crystallographic planar directions of anatase $\mathrm{TiO}_{2}$, respectively. According to Wulff construction, the proportion of exposed (101) and (001) facets in as-prepared samples can be calculated (Supplementary Fig. 1). The powder sample was characterized with $\sim 90 \%$ (101) facet, matching well with the thermodynamically stable (101) facet in anatase $\mathrm{TiO}_{2}$ predicted by Wulff construction $(\sim 94 \%)^{40}$. These particles gave an average face length of $3.8 \pm 0.5 \mathrm{~nm}$ (Fig. 1a and Supplementary Fig. 2a). According to the literature, when the concentration of HF is increased, it can increase the particle face length due to preferential adsorption of $\mathrm{F}$ to slow down its growth on this facet and hence the percentage of exposed (001) facet increases (Supplementary Table 1). Indeed, when $2 \mathrm{~mL} \mathrm{HF}$ was used in our case, an average face length of ca. $6.6 \pm 1.0 \mathrm{~nm}$ was achieved (Fig. 1c and Supplementary Fig. 2b) with a substantially higher proportion of (001) facet (Supplementary Table 1). By using $6 \mathrm{~mL} \mathrm{HF}$, the elongated face length of ca. $41.0 \pm 10.5 \mathrm{~nm}$ with a thickness of ca. $6.2 \pm 0.9 \mathrm{~nm}$ nanosheetlike F-(001) particles were obtained (Fig. 1e and Supplementary Fig. 2c). The percentages of exposed (101) and (001) facets of F(001) were estimated to be 24.6 and $75.4 \%$, respectively (Supplementary Table 1).

Figure $1 \mathrm{~b}, \mathrm{~d}, \mathrm{f}$ shows the corresponding ${ }^{31} \mathrm{P}$ MAS NMR spectra of TMP-adsorbed powder, F-(101) and F-(001) samples. This technique clearly gives distinctive fingerprint-like NMR patterns for $\mathrm{TiO}_{2}$ samples with various surface exposures/treatments. In general, the chemical shift of ${ }^{31} \mathrm{P}\left(\delta^{31} \mathrm{P}\right)$ in a range of -2 to -5 p.p.m. has been well attributed to the formation of a $\mathrm{TMPH}^{+}$ ionic complex formed when a TMP molecule is protonated (Brønsted acid site), while the $\delta^{31} \mathrm{P}$ of adsorbed TMP spans over a wide range ( -20 to -58 p.p.m.) when interacting with surface acidic site (LA site) with various Lewis acidity (the formation of TMP-LA) (Supplementary Note 1 and Supplementary Fig. 3) ${ }^{41}$. Accordingly, a stronger interaction of the surface acidic site with the basic TMP molecule is expected to give a more positive $\delta^{31} \mathrm{P}$ shift in the NMR spectrum, and the magnitude of this shift should depend on the adsorption energy/geometry on a particular facet and the TMP molecule ${ }^{7}$.

In order to compare these NMR peaks over the samples with different proportions of facets, ${ }^{31} \mathrm{P}$ MAS NMR peaks in the spectra of TMP-adsorbed $\mathrm{TiO}_{2}$ have been carefully deconvoluted. The powder sample shown in Fig. $1 \mathrm{~b}$ reveals no significant formation of $\mathrm{TMPH}^{+}$complex between -2 and -5 p.p.m. but TMP-LA complex with a main peak at $-36 \pm 1$ p.p.m. and a small shoulder at lower chemical shift $(-29 \pm 1$ p.p.m.) are clearly evident. The error of \pm 1 p.p.m. includes variations in facet dimension, defect concentration and measurement inaccuracy in the heterogeneous solid system. Notice that similar results in chemical shift values have been shown by Deng and co-workers ${ }^{37}$ over their titanium oxide. The major peak at -36 p.p.m. and the shoulder at -29 p.p.m. with the integrated area ratios of 84.8 and $15.2 \%$ respectively can be attributed to the interaction between TMP and surface five-coordinate Lewis acids: $\mathrm{Ti}^{4+}$ on the (101) facet as $\mathrm{Ti}_{5 \mathrm{C}}(101)$ and $\mathrm{Ti}^{4+}$ on $(001)$ as $\mathrm{Ti}_{5 \mathrm{C}}(001)$, respectively. This high proportion value of (101) facet in our powder sample matches with our earlier estimation using Wulff construction (89.8\%, Supplementary Table 1).

It has been observed that the adsorption energy of TMP molecule on the Lewis acid site shows a strong correlation with its NMR chemical shift value $7,41-43$. As shown in Fig. 2, our calculated adsorption energies of $\mathrm{TMP}$ on $\mathrm{Ti}_{5 \mathrm{C}}(001), \mathrm{Ti}_{5 \mathrm{C}}(101)$ and $\mathrm{Ti}_{5 \mathrm{C}} \mathrm{RC}($ reconstructed)(001) are obtained according to 

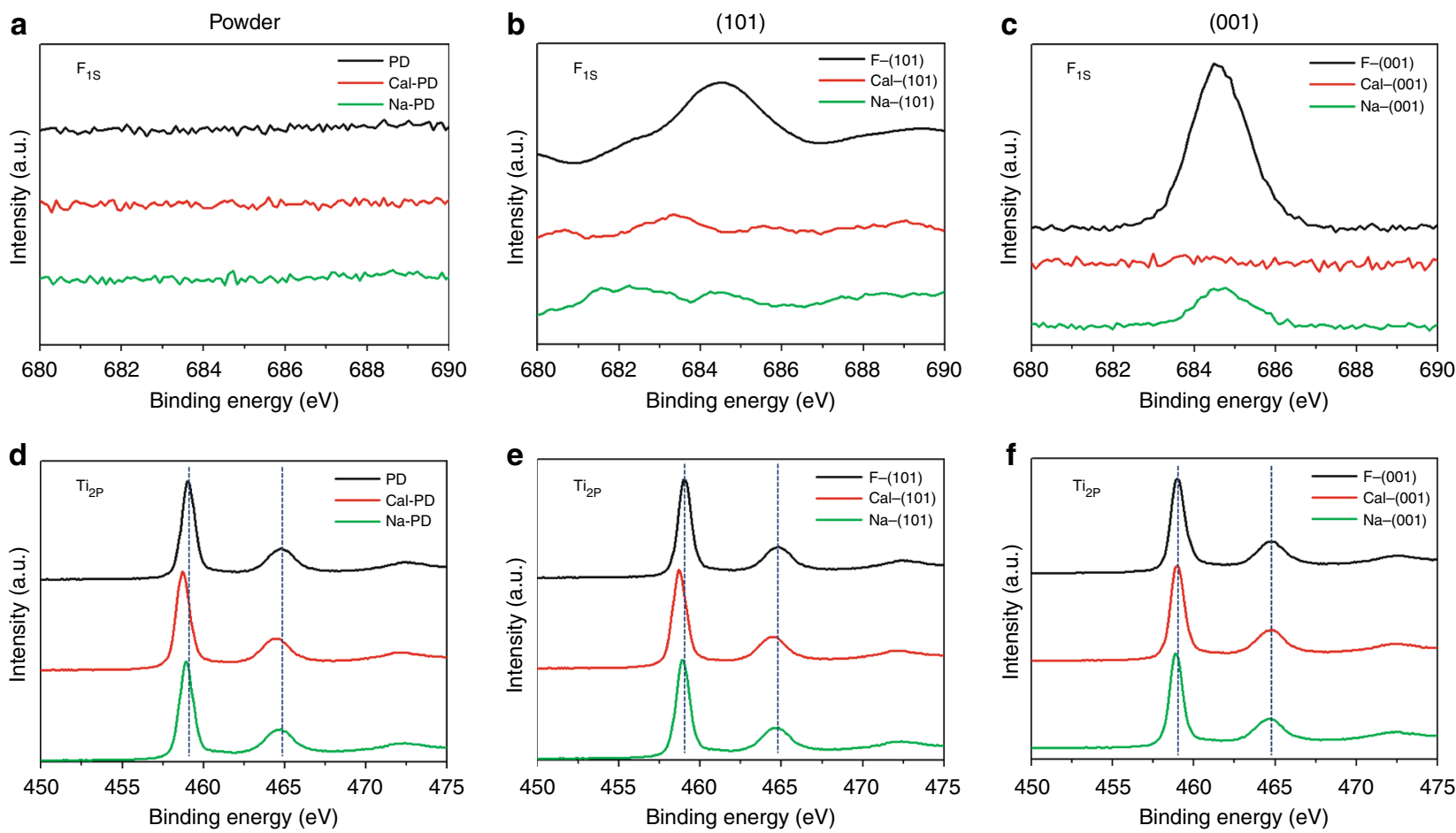

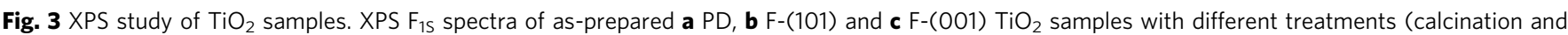
$\mathrm{NaOH}$ wash). $\mathbf{d}$ to $\mathbf{f}$ are the corresponding XPS $\mathrm{Ti}_{2 \mathrm{P}}$ spectra

computational models, respectively. Indeed, it is found that the calculated adsorption energy displays an excellent linear relationship with the experimental chemical shift value obtained (Supplementary Table 2 and Supplementary Fig. 4). Thus, this NMR technique is capable of differentiating facets of decreasing energy: $(001)>(101)>\operatorname{RC}(001)$ by their chemical shift values $(-29,-36$ and -50 p.p.m.), respectively. The relatively higher Brønsted acid signal of around -3 p.p.m. of F-(001) sample shown in Fig. If as compared to that of F-(101) sample shown in Fig. 1d again clearly indicates the preferential exposure of the (001) facet due to the increase in F concentration used.

On the other hand, some immediate questions remain to be answered from Fig. 1b, d, f. First, why did the resolved (101) peak of chemical shift $-31 \pm 1$ p.p.m. and (001) peak of $-22.5 \pm 1$ p.p.m. from the samples prepared with HF (i.e., F-(101) and F-(001)) show a consistent and significant 5 to 7 p.p.m. downshift from corresponding facets of -36 and -29 p.p.m. in the powder sample (no fluorine contamination), respectively? What is the nature of the peak with a chemical shift of $-42.5 \pm 1$ p.p.m., which was not seen over the PD sample? Why did the samples with HF treatment (i.e., F-(101) and F-(001)) show both Brønsted acid and Lewis acid signals but the as-prepared powder sample (PD) possessed mainly Lewis acid signal?

XPS, a technique to monitor element(s) on material surface, was used to analyse the surface compositions of these samples. As shown in Fig. $3 a-c$, the surface F on powder, F-(101) and F-(001) samples can be monitored by XPS as previously reported ${ }^{12}$. It is noted that $\mathrm{F}$ is apparently retained on the facets (101) and (001) when the samples were prepared with $F$ (Fig. $3 b, c)$ but the powder sample without previous exposure to $\mathrm{F}$ shows the total absence of $F_{1 S}$ signal (Fig. 3a). Despite the fact that XPS has been regarded as a surface-sensitive technique to atomic chemical states, it fails to give any chemical shift of $\mathrm{Ti}_{2 \mathrm{P}}$ in the presence of fluorine (Fig. $3 \mathrm{~d}-\mathrm{f}$ ). It is clear that the long electrons escape depth, greater than the outermost atomic layer (few atoms depth) of the sample, and this renders XPS unsuitable for surface studies in this context (detection limit $\sim 0.1 \%$ atom) and the monitoring of core electrons makes the binding energy of $\mathrm{Ti}$ also less sensitive to electronic effects from neighbouring $\mathrm{F}$ element(s). Auger electron spectroscopy is known to provide higher spatial resolution and the energy of ejected Auger electrons is more sensitive to the chemical environment compared to the core-level signals in XPS. However, as shown in Supplementary Fig. 5, no peak shift was also observed for five Ti LMM Auger signals (marked by dashed blue line). This indicates the change of chemical state of the outermost $\mathrm{Ti}$ atom is still averaged out during the collection of Auger electrons from the electron escape depth. In contrast, the ${ }^{31} \mathrm{P}$ NMR is much more surface sensitive to the electronic change imposed by this SDS.

The 5 to 7 p.p.m. downshift in values of the F-(101) and F-(001) samples from the corresponding facets of -36 p.p.m. $\mathrm{Ti}_{5 \mathrm{C}}(101)$ and -29 p.p.m. $\mathrm{Ti}_{5 \mathrm{C}}(001)$ in the powder sample to $-31 \pm 1$ p.p.m. and $-22.5 \pm 1$ p.p.m., respectively, are likely due to the presence of the electronic withdrawing effect of fluorine exerted to the $\mathrm{Ti}^{4+}$ on these two facets. Note that a surface-bound ligand molecule generates an electric dipole, while this intrinsic ligand dipole depends on its chemical structure and binding mode. For Lewis-basic ligands, the interfacial dipole points from the ligand toward the metal $\left(\mathrm{L}^{\delta-} \rightarrow \mathrm{M}^{\delta+}\right)$ and the largest ligandinduced downward shift of electronic energy levels is observed for halide ion ligands ${ }^{1}$. This electronic effect can be supported by our DFT calculation on a fluorinated (001) model. As shown in Supplementary Fig. 6, the calculated adsorption energy between TMP and $\mathrm{Ti}_{5 \mathrm{C}}$ on the clean (001) facet $(-1.20 \mathrm{eV}$, Fig. 2$)$ is greatly increased to $-1.76 \mathrm{eV}$ when the surrounding $\mathrm{Ti}_{5 \mathrm{C}}$ is fluorinated. According to the correlation plot between experimental $\delta^{31} \mathrm{P}$ and calculated adsorption energy in Supplementary Fig. 4, this can explain the downfield shift of F-(001) observed within experimental error. It is thought that a similar downfield shift for the F-(101) facet occurs upon the fluorination of surface $\mathrm{Ti}_{5 \mathrm{C}}$ at close proximity. The corresponding atomic ratios ( $\mathrm{Ti}, \mathrm{O}$ and $\mathrm{F}$ ) of $\mathrm{TiO}_{2}$ samples by XPS are summarized in Supplementary Table 3. 

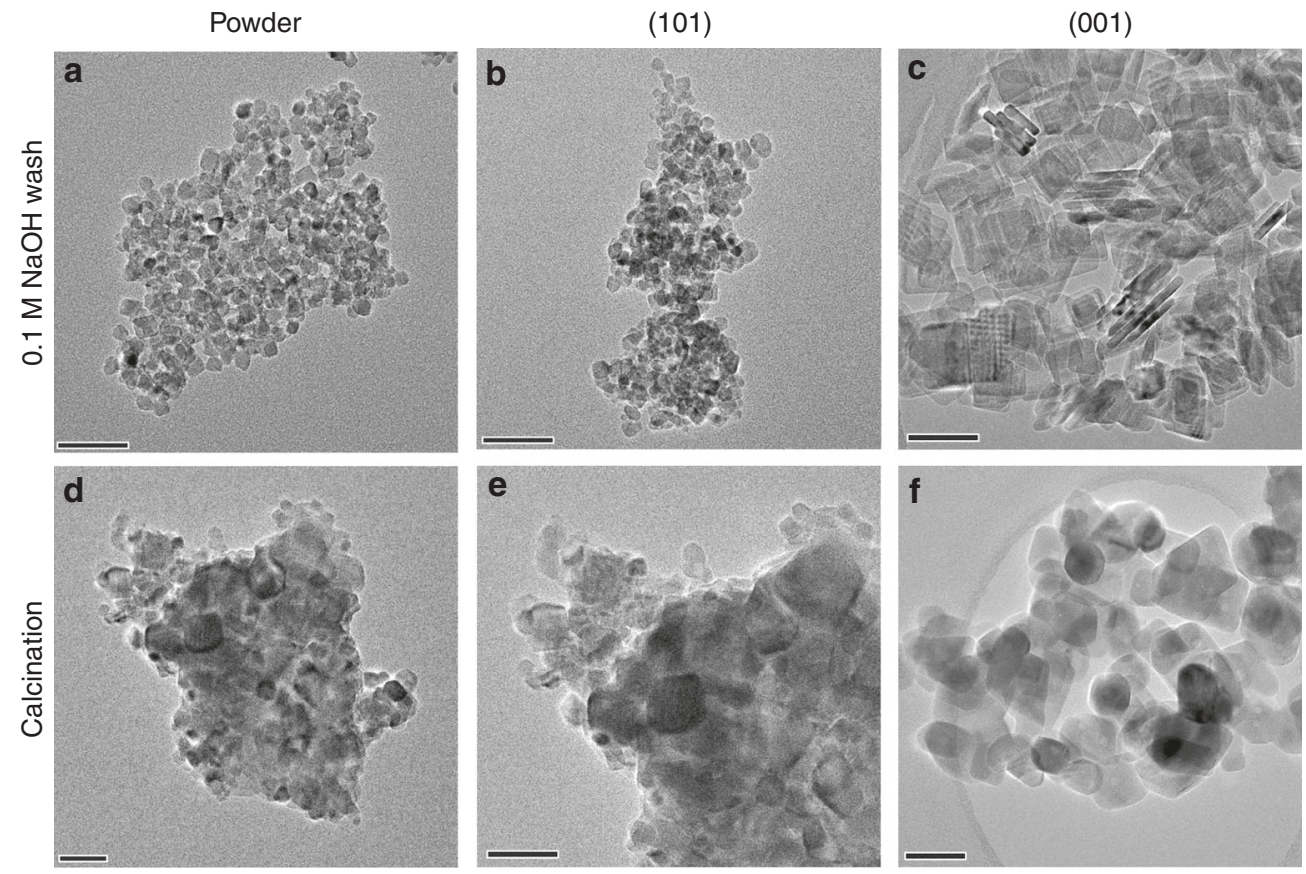

Fig. 4 Morphology of $\mathrm{TiO}_{2}$ samples after different treatments. TEM images of $\mathrm{TiO}_{2}$ samples after a-c $\mathrm{NaOH}$ wash: a $\mathrm{Na}-\mathrm{PD}, \mathbf{b} \mathrm{Na}-(101)$ and $\mathbf{c} \mathrm{Na}-(001)$ and d-f after calcination treatment: $\mathbf{d}$ Cal-PD, e Cal-(101) and $\mathbf{f}$ Cal-(001). The scale bar in all images is $50 \mathrm{~nm}$

The apparent lower $\mathrm{Ti}: \mathrm{O}$ values in the presence of $\mathrm{F}$ clearly suggest the formation of $\mathrm{V}_{\mathrm{o}}$ due to the strong electronic withdrawing effect of the surface fluorine groups. EPR (Supplementary Note 2, Supplementary Fig. 7 and Supplementary Tables 4 and 5) and Raman (Supplementary Note 3 and Supplementary Fig. 8) could also be used to indicate the electronic and structural changes of facets imposed by this surface impurity. Thus, such unstable (001) facets with F-containing surface oxygen vacancies could be hydrolysed (introduction of the $\mathrm{OH}$ functional group) during the treatment to account for the $-42.5 \pm 1$ p.p.m. peak observed (see later experiments). In addition, the evolution of Brønsted acidity over the fluorine-contaminated facets can be rationalized by the surface hydrogen bonding stabilization of protons by the fluorine. As a result from this study, one has to be very careful about the detainment of surface additive on a particular facet which can subtly affect its electronic, structural and geometric properties (acidity), and hence the evaluation of facet-dependent properties. Thus, post treatment to remove SDS before the study of facet-dependent properties is crucially important.

As stated, a $\mathrm{NaOH}$ wash ${ }^{13}, 15,17-19,24$ and calcination treatment $^{12,14-16,19,21,23,26,27,30}$ of F-treated samples at elevated temperature are the two commonly used methods in literature to remove surface $\mathrm{F}$ after the controlling of $\mathrm{TiO}_{2}$ particle morphology. The apparent remaining $\mathrm{F}$ signal on $\mathrm{Na}-(001)$ sample (cf. Cal-(001)) after $\mathrm{NaOH}$ (0.1 M) treatment shown in Fig. $3 \mathrm{c}$ clearly indicates that the $\mathrm{F}$ removal was not complete presumably due to the higher $F$ affinity for this energetic surface. In contrast, there is no F found in all calcined samples by the XPS (Fig. 3). However, this does not necessary suggest that calcination is a good method since high energetic facets may easily reconstruct to more stable surface at high temperature ${ }^{32,33}$. Moreover, as evidenced by TEM images (Fig. 4), calcination treatment causes severe particle aggregation for all three as-prepared PD, F-(101) and F-(001) while $\mathrm{NaOH}$ treatment does not lead to any observable aggregation and change in morphology. This result can be further supported by their corresponding XRD patterns (Supplementary Fig. 9). These two post-treatments according to our surface analysis could generate artefacts when facet-dependent properties are studied.

$\mathrm{NaOH}$ wash to remove surface fluorine. As $0.1 \mathrm{M} \mathrm{NaOH}$ has been widely adopted in literature to remove surface fluorine $^{13,15,17-19,24}$, ${ }^{31} \mathrm{P}$ MAS NMR spectra of TMP-adsorbed $\mathrm{Na}-\mathrm{PD}, \mathrm{Na}-(101)$ and $\mathrm{Na}-(001)$ were also collected and are deconvoluted in Fig. 5. In order to reduce the influence of $\mathrm{NaOH}$ on the measured chemical shift values, the samples after the $\mathrm{NaOH}$ treatment were repeatedly washed with deionized (DI) water. Notice that no significant residual $\mathrm{NaOH}$ was retained on these surfaces (Supplementary Note 4 and Supplementary Fig. 10). For samples prepared without HF (i.e., PD) (Fig. 5a), the $\mathrm{NaOH}$ treatment after rinsing with DI water clearly reduces the Lewis acidity of both $\mathrm{Ti}_{5 \mathrm{C}}(001)$ and $\mathrm{Ti}_{5 \mathrm{C}}(101)$ as $\delta^{31} \mathrm{P}$ shifts from -29 p.p.m. to $-35 \pm 1$ p.p.m. for $\mathrm{Ti}_{5 \mathrm{C}}(001)$ and from -36 p.p.m. to $-41 \pm 1$ p.p.m. for $\mathrm{Ti}_{5 \mathrm{C}}(101)$, respectively, for the surface mono-hydroxylation (cf. as-prepared PD, Fig. 1b). A shoulder appears at higher field ( $46 \pm 1$ p.p.m.) which is attributed to the formation of multi-hydroxylation $\mathrm{Ti}_{5 c} \mathrm{O}_{5-x}(\mathrm{OH})_{x}$ with $x>2$ during the extensive hydrolysis by the $\mathrm{NaOH}$ treatment at high concentration (Supplementary Fig. 11) ${ }^{44,45}$. However, for F-treated samples (i.e., F-(101) or F-(001)), a different Lewis acid distribution is obtained after the same treatment (Fig. 5b, c). The conversion of residual $\mathrm{F}-\mathrm{Ti}$ to $\mathrm{HO}-\mathrm{Ti}$ on high energetic facets, particularly on (001), by $\mathrm{OH}$ - exchange in the $\mathrm{NaOH}$ wash can give a very different electron density of $\mathrm{Ti}_{5 \mathrm{C}}$ atoms compared to surface Ti without the $\mathrm{F}$. The surface chemical functionalization by $\mathrm{OH}$ shifts the $\delta^{31} \mathrm{P}$ of TMP-adsorbed $\mathrm{F}^{-T i_{5 \mathrm{C}}}(001) / \mathrm{F}-\mathrm{Ti}_{5 \mathrm{C}}(101)$ from $-22.5 \pm 1 /-31 \pm 1$ p.p.m. (Fig. $1 d, f$ ) to $-28 \pm 1 /-36.5 \pm 1$ p. p.m. (Fig. 5b, c). In addition, the typical Brønsted acid signals (the formation of $\mathrm{TMPH}^{+}$) between -2 and -5 p.p.m. clearly indicate that there is a residue $\mathrm{F}$ left on both $\mathrm{Na}-(101)$ and $\mathrm{Na}-(001)$ sample surfaces (Fig. 5b, c). On the other hand, the total Fdepleted surfaces of $-35 \pm 1$ p.p.m. for $\mathrm{Ti}_{5 \mathrm{C}}(001)$ and $-41 \pm 1$ p.p. $\mathrm{m}$. for $\mathrm{Ti}_{5 \mathrm{C}}(101)$ are expected to re-emerge since $\mathrm{NaOH}$ is capable of removing some surface F. The former peak ( -35 p.p.m.) is 

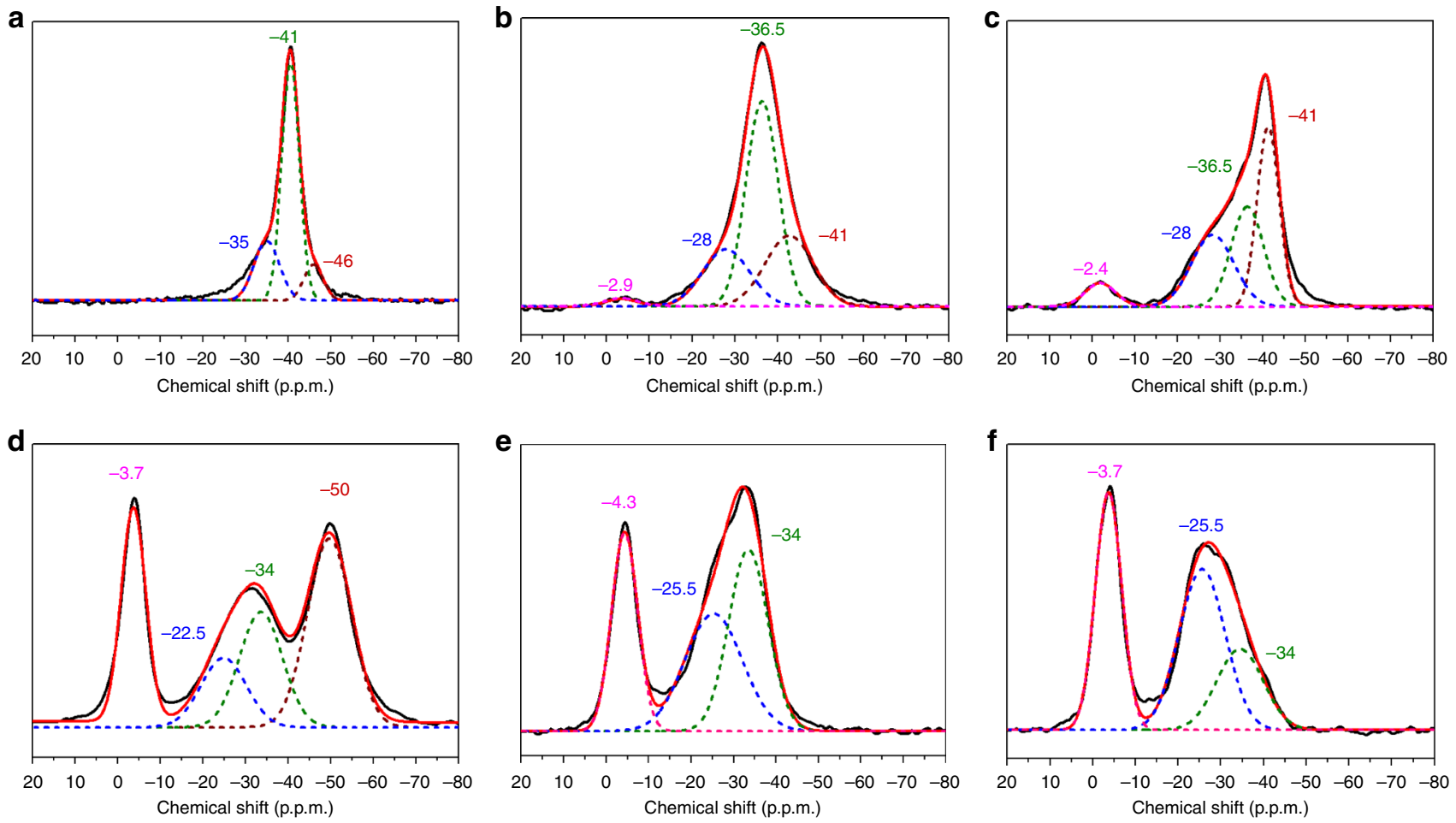

Fig. $5 \mathrm{NMR}$ results of $\mathrm{NaOH}$-washed $\mathrm{TiO}_{2}$ samples and their following sulfation treatment. The ${ }^{31} \mathrm{P}$ MAS NMR spectral deconvolution of TMP-adsorbed $\mathrm{TiO}_{2}$ samples treated with $0.1 \mathrm{M} \mathrm{NaOH}$ : a Na-PD, b Na-(101) and c Na-(001) and their following sulfation treatment: d S-Na-PD, e S-Na-(101) and f S-Na-(001)

unfortunately mixed with the $\mathrm{OH}$-shifted $\mathrm{F}^{-\mathrm{Ti}_{5 \mathrm{C}}}(001)$ peak (-36.5 p.p.m.) but the prominent at $-41 \pm 1$ p.p.m. can be attributed to the reformation of $\mathrm{Ti}_{5 \mathrm{C}}(101)$ (Supplementary Fig. 11) during its surface hydrolysis by the $\mathrm{NaOH}$ treatment.

Post-calcination to remove surface fluorine. Recently, the heatinduced aggregation of sheet-like anatase $\mathrm{TiO}_{2}$ has been shown to occur preferentially along the [001] crystallographic direction, driven by the minimization of surface energy 46,47 . According to Yang et al. ${ }^{46}$, surface Ti-F groups are totally hydrolysed during calcination in humid air at high temperature (endothermic reaction) (Supplementary Fig. 12). In this case, the exposed surfaces of sheet-like anatase $\mathrm{TiO}_{2}$ are also mainly covered by -OH groups. As the (001) facet has a higher surface energy than the (101) facet, the (001) interfaces between adjacent nanosheets would be eliminated through the condensation of $\mathrm{Ti}-\mathrm{OH}$ groups (formation of Ti-O-Ti linkages) at high temperature and therefore the (101) facet becomes dominant (Supplementary Fig. 12). According to our XPS (Fig. 3), TEM (Fig. 4)/high-resolution TEM (Supplementary Fig. 13) and XRD (Supplementary Fig. 9) data, the post calcination is effective to remove F from (001) and (101) surfaces, which can clearly induce aggregation.

The ${ }^{31} \mathrm{P}$ MAS NMR spectra of TMP-adsorbed Cal-PD and Cal-(001) were deconvoluted and are shown in Supplementary Fig. 14a, b. It is noted that calcination in humid air pushes the $\delta^{31} \mathrm{P}$ of $\mathrm{Ti}_{5 \mathrm{C}}(001) / \mathrm{Ti}_{5 \mathrm{C}}(101)$ of $\mathrm{PD}$ from $-29 /-36$ to $-35 \pm 1 /-41 \pm 1$ p.p.m. due to the surface mono-hydroxylation as mentioned previously (cf. as-prepared PD, Fig. 1b). Interestingly, Supplementary Fig. 14a shows a new but characteristic signal of Cal-PD at $-50 \pm 1 \mathrm{~nm}$. According to our DFT calculation (i.e., -50 p.p.m., Fig. 2), this is due to the adsorption of TMP on $\mathrm{Ti}_{5 \mathrm{C}}$ on $(1 \times 4)$ the reconstructed $(001)$ facet $\left(\mathrm{Ti}_{5 \mathrm{C}} \mathrm{RC}\right.$ (001)). Thus, this peak can be used as a diagnostic peak to indicate reconstruction/aggregation induced by calcination.
However, for $\mathrm{F}-(001)$, the same chemical shifts for $\mathrm{Ti}_{5 \mathrm{C}}(001) /$ $\operatorname{Ti}_{5 \mathrm{C}}(101)$ (i.e., $-35 \pm 1 /-41 \pm 1$ p.p.m.) indicative of the monohydroxylation of the surfaces with no residual $\mathrm{F}$ can be obtained by calcination (Supplementary Fig. 14b) but not for the $\mathrm{NaOH}$ wash at room temperature, where retention of $\mathrm{F}$ on $\mathrm{Ti}_{5 \mathrm{C}}(001) / \mathrm{F}$ $\mathrm{Ti}_{5 \mathrm{C}}(101)$ is evident $(-28 \pm 1 /-36.5 \pm 1$ p.p.m., see Fig. $5 \mathrm{c})$. The large signal of Cal-(001) at $-45 \pm 1$ p.p.m. (Supplementary Fig. 14b) can be assigned to the metastable $(1 \times 3)$ and $(1 \times 5)$ (001) facets originating from the dominant high energy but unstable $(1 \times 1)(001)$ facet before their further transformation to the more stable reconstructed $(1 \times 4)$ structure $(\text { at }-50 \text { p.p.m. })^{33}$. By comparing the area of deconvoluted peaks of Cal-(001), we are able to show an $\sim 80 \%$ of (001) facet that had been reconstructed during the calcination.

Surface sulfation. As stated, surface functionalization could severely alter facet properties but this issue has not been much addressed in previous literature. The sulfation of $\mathrm{TiO}_{2}$ facets was therefore investigated after the calcination or $\mathrm{NaOH}$ wash with a prolonged period of time to ensure no $\mathrm{F}$ remained on the surfaces. The ${ }^{31} \mathrm{P}$ MAS NMR result for calcined samples after sulfation treatment (i.e., S-Cal-PD and S-Cal-(001)) are shown separately in Supplementary Fig. 14c, d. As calcination treatment causes severe aggregation and unavoidable facet reconstruction, we herein focus on the sulfation of $\mathrm{NaOH}$-washed samples. For F-(101) and F-(001) samples, Brønsted acid (Ti-O(H)-Ti) sites removed by $\mathrm{NaOH}$ wash can clearly be reintroduced by a sulfation step with a large and distinctive peak around -4 p.p.m. (Fig. 5e, f). It is interesting to note that two new and characteristic strong Lewis acid peaks due to TMP adsorption on sulfated surfaces, namely $-25.5 \pm 1$ and $-34 \pm 1$ p.p.m., are introduced. It is postulated these two new Lewis acid sites are arisen from the $\mathrm{Ti}^{4+}$ modified directly by $\mathrm{SO}_{4}$ at high surface coverage during the extensive sulfation but further verifications are required. Their 
a

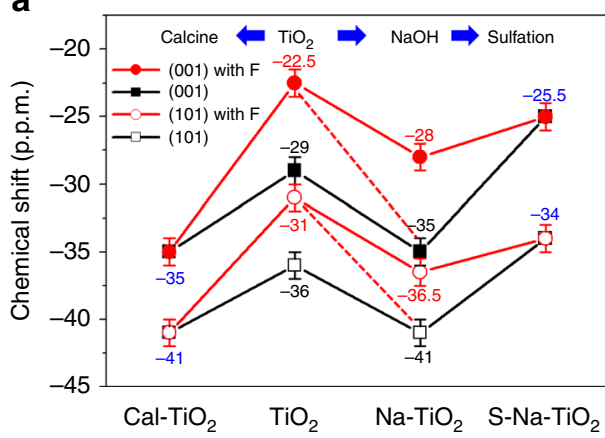

b

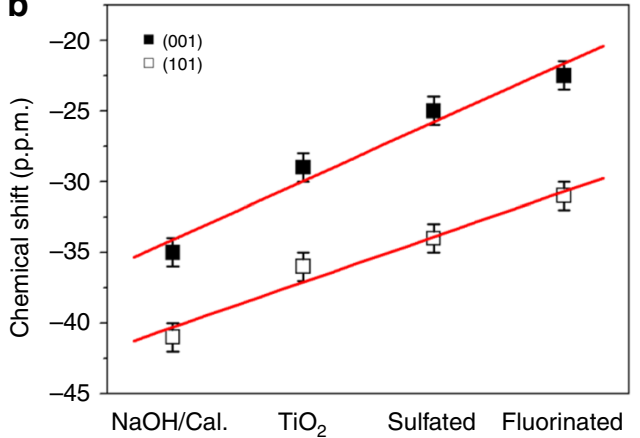

C
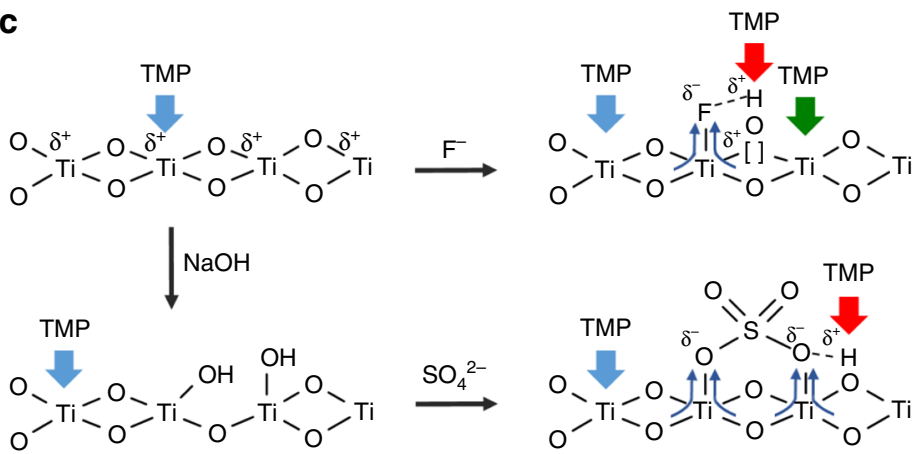

$\stackrel{\mathrm{SO}_{4}^{2-}}{\longrightarrow}$

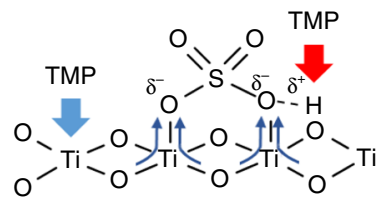

Fig. 6 Interaction between TMP and surface features on $\mathrm{TiO}_{2}$ facet with various treatments/modifications. a The $\delta^{31} \mathrm{P}$ of $\mathrm{TMP}$-adsorbed $\mathrm{Ti}_{5 \mathrm{C}}$ on (001)/(101) facets (from PD) and on F-modified (001)/(101) facets (from F-(001) and F-(101)) with different treatments/modifications. b The summary of $\delta^{31} \mathrm{P}$ of $\mathrm{TMP}$-adsorbed $\mathrm{Ti}_{5 \mathrm{C}}$ on $(001) /(101)$ facets with different treatments (calcination/ $\mathrm{NaOH}$ wash) and modification (sulfate/fluorine). The error bar in a, $\mathbf{b}$ is \pm 1 p.p.m. $\mathbf{c}$ Illustration of interaction between TMP and surface features on $\mathrm{TiO}_{2}$ facet with various treatments/modifications

strengths (peak shifts) are sensitive to the particular facets and their relative concentrations (peak areas) also depend on the relative proportions of (101) and (001) in the samples. For instance, S-Na-(101) with the preferentially exposed (101) facet shows a stronger signal at $-34 \pm 1$ p.p.m. (Fig. 5e), while for $\mathrm{S}-\mathrm{Na}-(001)$ with the preferentially exposed (001) facet gives a more dominant signal at $-25.5 \pm 1$ p.p.m. (Fig. $5 f$ ). For the PD samples after the extensive hydrolysis during treatments, surface multi-hydroxylation is expected to give a peak at $-50 \pm 1$ p.p.m., as well as the two Lewis acid peaks of $-25.5 \pm 1$ and $-34 \pm 1$ p.p.m. (Fig. 5d).

Qualitative and quantitative analysis. Figure 6 and Table 2 summarize qualitative (chemical shift) and quantitative (peak area) information of deconvoluted peaks in TMP-adsorbed $\mathrm{TiO}_{2}{ }^{31} \mathrm{P}$ MAS NMR spectra under various treatments. First, Fig. 6a shows that the adsorption of TMP on Lewis acid centres of the higher energy (001) facet gives lower chemical shift value $(-29 \pm 1$ p.p.m.) than that of the more stable (101) facet $(-36 \pm 1$ p.p.m.) in our samples. F-treatment of the $(001)$ and (101) facets can clearly enhance the Lewis acidity, shifting them to $-22.5 \pm 1$ and $-31 \pm 1$ p.p.m., respectively. This surface group with the highest electron withdrawing ability can also generate oxygen vacancies, and hence activating the surfaces substantially. However, it is clear from our study that calcination in humid air at elevated temperature can indeed remove the surface $F$ groups by introducing hydroxylation to the surfaces. Replacing $\mathrm{F}$, with a strong withdrawing propensity, by much weaker surface $\mathrm{OH}$ group will render the upshifts to $-35 \pm 1$ p.p.m. for the (001) facet and $-41 \pm 1$ p.p.m. for the (101) facet due to mono-hydroxylation as mentioned previously (multi-hydroxylation can further upshift the $\delta^{31} \mathrm{P}$ of the adsorbed TMP). However, the high coverage of $\mathrm{OH}$ particularly on (001) facet at elevated temperature during calcination can concomitantly lead to severe aggregation. For a prolonged period of time, this can lead to stepwise reconstruction of $(1 \times 1)(001)$ facets to the more stable reconstructed $(1 \times 4)$ structure (at -50 p.p.m.). On the other hand, the replacement of surface $\mathrm{F}$ with $\mathrm{OH}$ using a $\mathrm{NaOH}$ wash at room temperature can also achieve the $\mathrm{F}$ removal but retain the integrity of desired facets and prevent significant aggregation and reconstruction, giving the same shift values of $-35 \pm 1$ p.p.m. for the (001) facet and $-41 \pm 1$ p.p.m. for the (101) facet. However, our TMP-adsorption technique supported by other characterizations (XPS, EPR, Raman) show clearly that a $\mathrm{NaOH}$ wash at different times and concentrations at room temperature may not be sufficient to remove all surface $\mathrm{F}$ from the $\mathrm{TiO}_{2}$. If $\mathrm{F}$ is retained together with the mono-hydroxylated $\mathrm{OH}$, it can give two new $\delta^{31} \mathrm{P}$ values (different Lewis acid strengths) of TMP-adsorbed $\mathrm{F}-\mathrm{Ti}_{5 \mathrm{c}}(001) / \mathrm{F}-\mathrm{Ti}_{5 \mathrm{c}}(101)$ of $-28 \pm 1 /-36.5 \pm 1$ p.p.m. (Fig. 6a). In addition, two strong Brønsted acid strengths (the formation of $\mathrm{TMPH}^{+}$) at -2.9 and -2.4 p.p.m. (Fig. 5b, c) are also achieved due to the proton stabilization by residual $\mathrm{F}$ left on both $\mathrm{Na}-(101)$ and $\mathrm{Na}$-(001) sample surfaces. Upon extended sulfation after the samples are calcined (Supplementary Fig. 14c, d) or $\mathrm{NaOH}$ (Fig. 5e, f) washed to replace all $\mathrm{F}$ with $\mathrm{SO}_{4}$, weaker Brønsted acid strengths between -3.7 and -4.7 p.p.m. with protons influenced by neighbour $\mathrm{SO}_{4}$ are obtained. In terms of Lewis acid alteration, $-25.5 \pm 1$ p.p.m. from sulfation of $(001)$ and $-34 \pm 1$ p.p.m. of (101) facets can be tuned. Figure $6 \mathrm{~b}$ shows the highly sensitive $\delta^{31} \mathrm{P}$ value towards increasing electron withdrawing ability to the surface $\mathrm{Ti}^{4+}$ from $\mathrm{OH}<-\mathrm{O}-<\mathrm{SO}_{4}<\mathrm{F}$ which can provide finetuning of Lewis acid and Brønsted acid sites on $\mathrm{TiO}_{2}$ facets by these surface additives. Figure $6 \mathrm{c}$ summarizes the anticipated molecular interactions between TMP and surface features on $\mathrm{TiO}_{2}$ with various treatments/modifications. In addition, the TMP-assisted NMR technique displays quantitative assessments on the surface speciation (Table 2). The total adsorbed TMP over 
Table 2 Qualitative and quantitative results of all surface features on $\mathrm{TiO}_{2}$ facet with various treatments and modifications

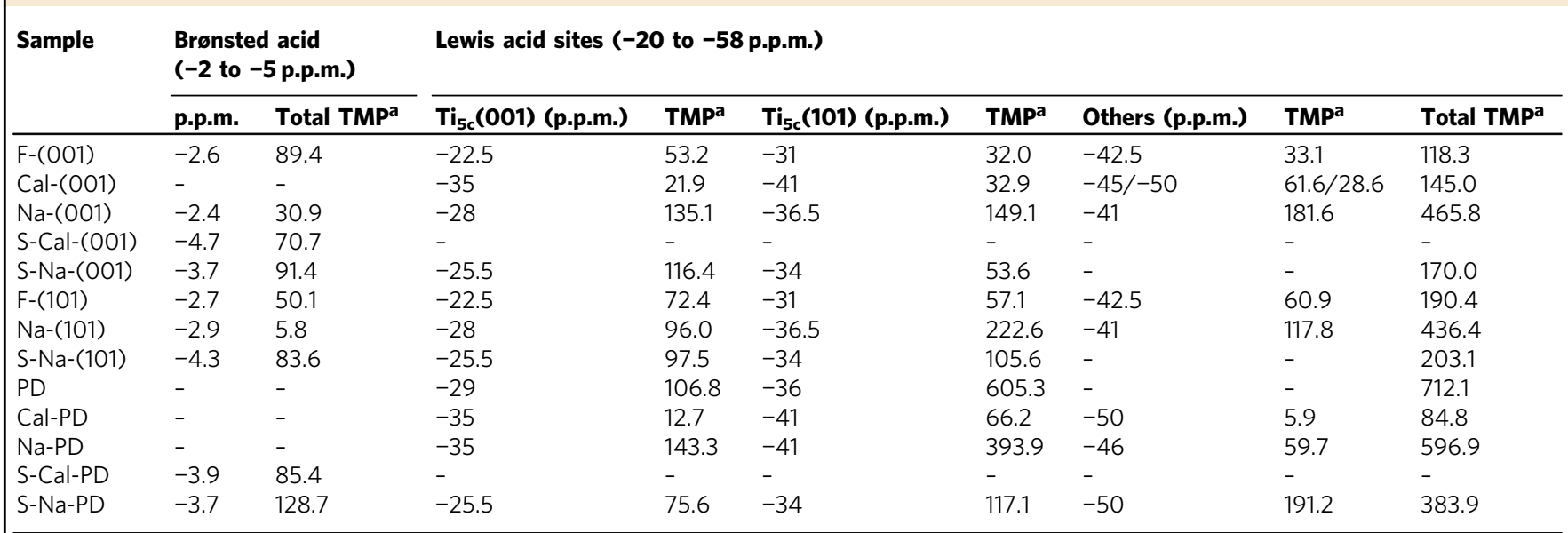

Summarizes the qualitative (chemical shift) and quantitative (peak area) of each deconvoluted peak in the region of Brønsted acid site ( -2 to -5 p.p.m.) and Lewis acid site ( -20 to -58 p.p.m.). The concentration of adsorbed TMP on each site was calculated according to corresponding peak area ( ${ }^{\mathrm{a} A d s o r b e d ~ T M P ~ m o l e c u l e s ~ i n ~} \mu \mathrm{mol} / \mathrm{g}$ )

different samples also shows strong correlations with the total BET (Brunauer-Emmett-Teller) surface areas of the samples (BET of S-Na-PD $=107.0 \mathrm{~m}^{2} / \mathrm{g}, \mathrm{S}-\mathrm{Na}-(101)=77.3 \mathrm{~m}^{2} / \mathrm{g}$ and $\mathrm{S}-$ $\mathrm{Na}-(001)=67.4 \mathrm{~m}^{2} / \mathrm{g}$ (Supplementary Table 6), which is proportional to their total TMP/g (both Brønsted acid and Lewis acid from Table 2) uptakes of 512.6, 286.7 and $261.4 \mu \mathrm{mol} / \mathrm{g}$, respectively). Typically, this technique shows a better surface sensitivity; for example, $5.8 \mu \mathrm{mol} / \mathrm{g}$ Brønsted acid stabilized by F on Na-(101) is detectable by the NMR (Table 2) but no corresponding F signal is observed by XPS (Fig. 3b). It is particularly noted that XPS fails to reveal the change in $\mathrm{Ti}_{2 \mathrm{P}}$ electronic state with or without fluorine (Fig. $3 \mathrm{~d}-\mathrm{f}$ ) but ${ }^{31} \mathrm{P}$ MAS NMR can lead to their detailed assessments (Table 2).

Catalytic testing. It is well accepted that photocatalytic activity is highly dependent on particular facets of transition metal oxide crystals. Pioneered by Han et al. ${ }^{13}$ and Yang et al. ${ }^{14}$, using sheet-like $\mathrm{TiO}_{2}$ with a higher percentage of reactive (001) facets was proven to exhibit higher photocatalytic activity in aqueous medium (cf., P25). Both of the above reports also showed that the photocatalytic efficiency of the $\mathrm{TiO}_{2}$ nanosheets can be further improved after removing surface fluorine either by calcination $^{12,14}$ or $\mathrm{NaOH}$ wash $^{13}$. However, the changes in the quantity and quality of active sites on this (001) facet upon the above treatments were still unable to offer detailed analysis and assessment due to the lack of appropriate characterization techniques ${ }^{12-31}$. To illustrate the importance of acquiring the delicate surface electronic and structural changes imposed by a particular facet and surface chemical modifier, the influence of various treatments to $\mathrm{TiO}_{2}$ surface features were monitored (Table 2). The photocatalytic decomposition rate of malachite green (MG) upon F-(001), Cal-(001) and $\mathrm{Na-(001)}$ in static solution was therefore studied to reassess the surface structure-photocatalytic activity relationships ${ }^{13-24}$. Corresponding results are shown in Fig. 7a. For samples with the preferential exposure of (001) facet, the $\mathrm{NaOH}$-washed $\mathrm{F}$-(001) sample (i.e., Na-(001)) shows the highest photo-decomposition efficiency while the sample without any treatment (i.e., F-(001)) shows the lowest efficiency (activity: Na-(001)>Cal- $(001)>\mathrm{F}-(001))$. This result indicates that the key factors are neither crystallinity nor surface area. This is because F-(001) possesses similar crystallinity (Supplementary Fig. 9)/surface area (Supplementary Table 6) to $\mathrm{Na}-(001)$ and has a higher surface area than Cal-(001) but shows the lowest activity. The role of surface defect such as $V_{o}$ is also proved to be minor as both XPS Ti to O ratio (Supplementary Table 3) and quantitative EPR signal at g 2.0 (Supplementary Table 4) suggest the $\mathrm{V}_{\mathrm{o}}$ concentration is in a reverse order: $\mathrm{F}-(001)>$ Cal-(001) $>\mathrm{Na}-(001)$. There is also no significant change in their measured bandgap values (Supplementary Fig. 15), which is commonly reported to be one of the important factors in photocatalysis ${ }^{16}$. According to our NMR results, the possibility of Brønsted acid playing a key role is also ruled out as the Cal-(001) with no detectable Brønsted acid exhibits a higher percentage of MG degradation rate than F-(001), which has the highest Brønsted acid concentration in solution. The acid strength of Lewis acid also does not seem to play the key role in this reaction either as F-(001) with the strongest Lewis acid site (at -22 p.p. m., Fig. 7b) exhibits the lowest activity. In this case, the total Lewis acid concentration $(465.8 \mu \mathrm{mol} / \mathrm{g}$ of $\mathrm{Na}-(001)>145 \mu \mathrm{mol} / \mathrm{g}$ of Cal-(001) $>118.3 \mu \mathrm{mol} / \mathrm{g}$ of F-(001)) appears to be the primary cause for this activity (Fig. 7a) as it is proportional to the rate and yield of photodecomposition of the MG molecules. As the fluorine binds strongly to the surface $\mathrm{Ti}$ atom (blocking the Lewis acid site) on (001) facet, it is not surprising that its removal by $\mathrm{NaOH}$ wash in the $\mathrm{Na}-(001)$ sample with similar particle size (cf., F-(001)) has quadrupled the Lewis acid surface concentration. Even though severe aggregation has taken place over the calcined Cal-(001) sample, it still possesses a higher Lewis acid concentration than that of F-(001). A similar conclusion can also be drawn for samples with preferentially exposed (101) facets (i.e., PD, see Supplementary Note 5 and Supplementary Fig. 16 also for commercial P25). This indicates that the surface feature and surface area are the key factors for the observed difference in the decomposition rates. This result can be supported by the observation of our previous study which found that the small degree of adsorption of dye molecules due to limited Lewis acid sites was the rate-determining step for photocatalytic reaction in the $\mathrm{Nb}_{2} \mathrm{O}_{5}$ case in static solution ${ }^{48}$. However, the photocatalytic reaction carried out in the flowing air seems to depend on Lewis acid strength more than the total Lewis acid concentration. Xiang et al. ${ }^{19}$ reported that $\mathrm{F}_{-} \mathrm{TiO}_{2}$ gives better activity than treated $\mathrm{TiO}_{2}$ (i.e., $\mathrm{Cal}-\mathrm{TiO}_{2}$ and $\mathrm{Na}-\mathrm{TiO}_{2}$ ) for the photocatalytic degradation of acetone in flowing air. They suggested the strong electron withdrawing ability of the surface Ti-F groups reduces the recombination of photo-generated electrons and holes and enhances the photocatalytic activity. However, according to our result, we believe the adsorption dynamic of acetone in flowing 
a

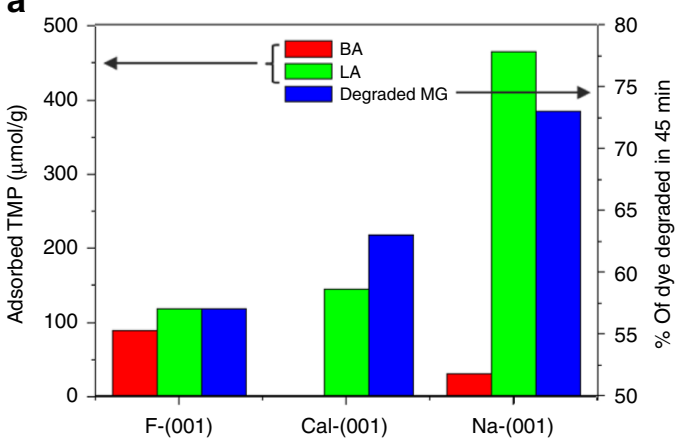

C

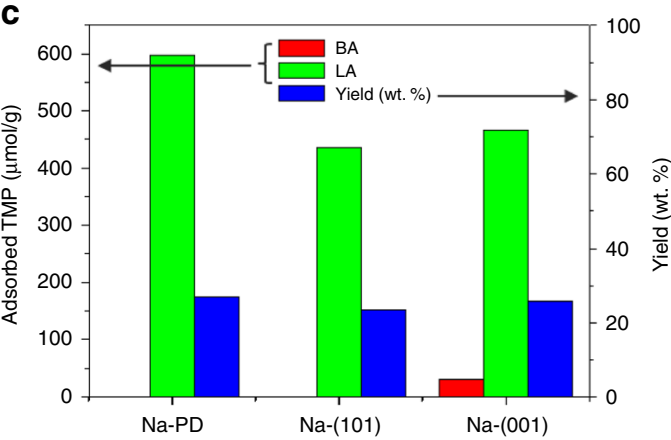

e

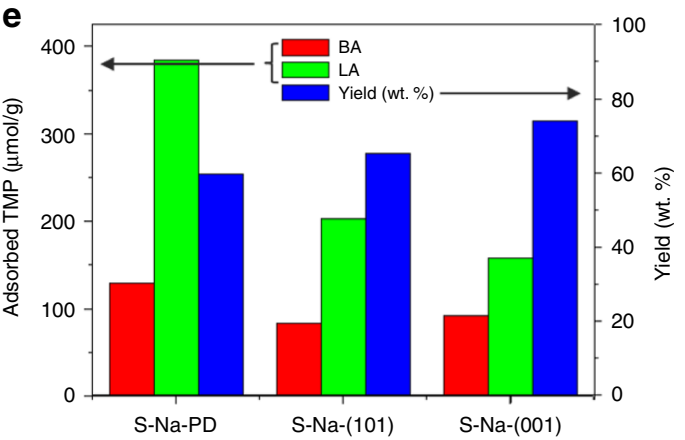

b

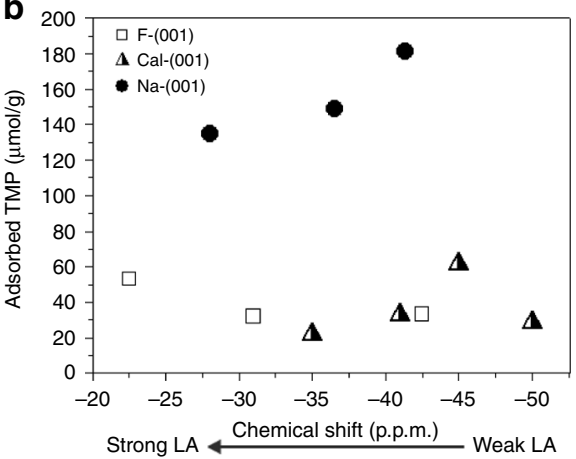

d

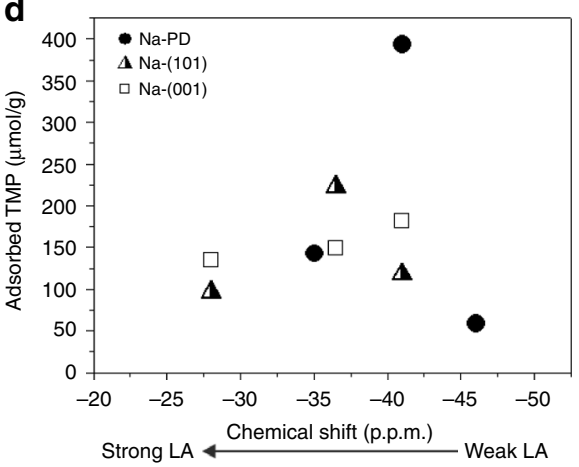

f

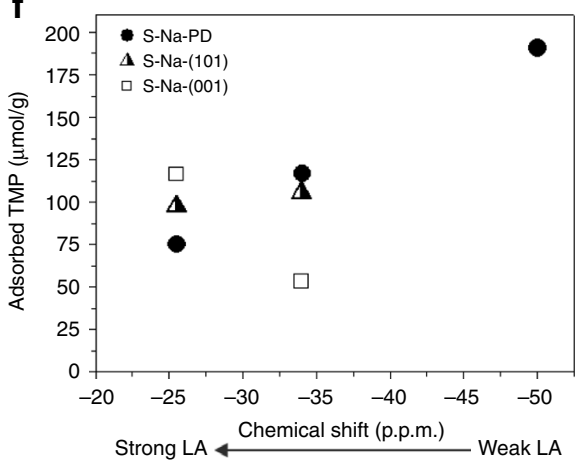

Fig. $7 \mathrm{TiO}_{2}$ catalytic activity and the distribution of corresponding active sites. Comparison of Brønsted acid (BA) and Lewis acid (LA) for a photocatalytic decomposition rate of malachite green (MG) dye in 45 min over F-(001) samples with various treatments. Pechmann condensation activity over c $\mathrm{NaOH}$ treated samples and $\mathbf{d}$ further sulfated samples. $\mathbf{b}, \mathbf{d}, \mathbf{f}$ are corresponding concentrations of Lewis acid sites of tested samples with different strengths

air is very different from that of dye in static water and can only be efficiently adsorbed from the gas phase by the stronger Lewis acid site of $\mathrm{F}^{-\mathrm{TiO}_{2}}$ (e.g., -22 p.p.m., Fig. 7b) before the photodecomposition. Thus, the stronger nature of Lewis acid site of $\mathrm{F}_{-}-\mathrm{TiO}_{2}$ renders higher activity than that of the treated $\mathrm{TiO}_{2}$ (i.e., $\mathrm{Cal}-\mathrm{TiO}_{2}$ and $\mathrm{Na}-\mathrm{TiO}_{2}$ ) in the gas phase degradation of acetone in air.

To demonstrate the importance of assessing the electronic and structural states on facets both qualitatively and quantitatively in heterogeneous catalysis, the catalytic performances of $\mathrm{Na}-\mathrm{PD}, \mathrm{Na}-(101), \mathrm{Na}-(001)$ and corresponding sulfation samples (i.e., S-Na-PD, S-Na-(101) and S-Na-(001)) for the catalytic Pechmann condensation reaction using phloroglucinol and ethyl acetoacetate as starting reagents were carried out and the results are shown in Fig. $7 \mathrm{c}$, e. In view of the Pechmann reaction, the condensation of phenol and $\beta$-keto ester proceeds through transesterification followed by intramolecular hydroalkylation and dehydration ${ }^{49}$. As shown in Fig. 7c, the yield of 5,7-dihydroxy-4-methyl coumarin of $\mathrm{Na}-\mathrm{PD}$ $(27.13$ wt.\%) $\geq \mathrm{Na}-(001)(25.86$ wt.\%) $=\mathrm{Na}-(101)(23.53$ wt.\%) in solution shows a slight correlation with total Lewis acid concentration but both Lewis acid strength (Fig. 7d) and the trace Brønsted acid of $\mathrm{Na}$-(001) show almost no significant influence (total concentration of Lewis acid is more important than its strength in solution catalysis as previously discussed). However, the yield is almost tripled when using corresponding sulfation samples in the order of S-Na- $(001)$ (73.87 wt.\%)>S-Na-(101) (65.16 wt.\%)>S-Na-PD (59.68 wt.\%) (Fig. 7e). This huge increase in yield for all the three samples could be easily mistakenly attributed to the introduction of weak Brønsted acid by sulfate modification at the expense of Lewis acid sites (see Fig. 7c, e). However, S-Na-PD with the highest total concentrations in both Brønsted acid and Lewis acid shows the lowest yield among the three samples and similar Brønsted acid strength is actually obtained for all the three samples. Thus, Brønsted acid does not seem to be the main site for this catalysed reaction. Notice that the sulfate modification of S-Na-PD, S-Na-(101) and S-Na-(001) also concomitantly introduces a new Lewis acid site of much greater strength than unmodified samples (Fig. 7f). The strength of this newly generated Lewis acid site at $\sim-25$ p.p.m. ( $\mathrm{Ti}^{4+}$ modified directly by the neighbours $\mathrm{SO}_{4}$ and $\mathrm{OH}$, see Fig. $6 \mathrm{c}$ ) is found 

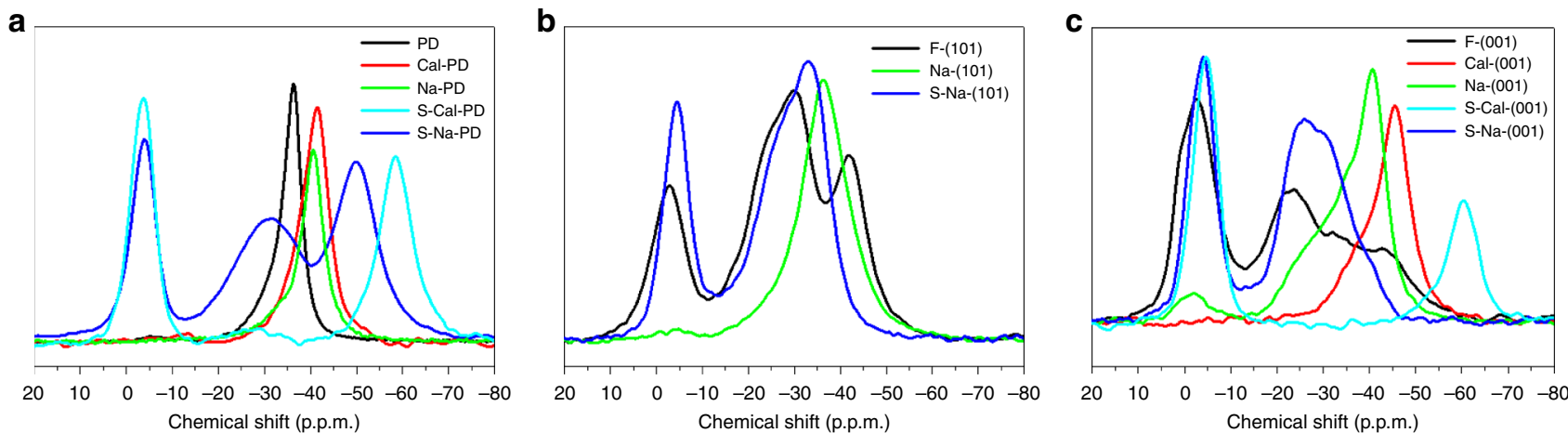

Fig. 8 Superposition of ${ }^{31} \mathrm{P}$ NMR spectra of $\mathrm{TMP}$-adsorbed $\mathrm{TiO}_{2}$ samples. Summary of the electronic effect (chemical shift) imposed by different adsorbates during sequential treatments/modifications on a PD, b F-(101) and c F-(001) with various exposed facets

to be comparable to sulfated $/ \mathrm{BF}_{3}$-modified metal oxides $\left(\mathrm{SO}_{4}{ }^{2-} / \mathrm{ZrO}_{2}{ }^{50}, \mathrm{BF}_{3} / \mathrm{Al}_{2} \mathrm{O}_{3}{ }^{51}\right)$ which is characteristic of super Lewis acidity. This Lewis acid concentration is clearly found to be in the order of S-Na-(001)>S-Na-(101)>S-Na-PD (Fig. 7f) in accordance with the order of their product yield, which undoubtedly suggests that the specific concentration of this new-generated super acid site is responsible for catalysing this reaction in solution accordingly.

\section{Discussion}

The nanometre dimensions and intrinsic heterogeneity of NPs (each particle typically exposes several facets with different patterns of surface atoms) makes the experimental study of surfaces challenging. Traditional surface tools such as XPS, EPR and Raman spectroscopy have been widely employed to bridge facet-controlled NPs and their corresponding facet-dependent performances. However, as demonstrated in this study (using $\mathrm{TiO}_{2}$ ), these techniques (XPS: Fig. 3; EPR: Supplementary Fig. 7; Raman: Supplementary Fig. 8) can only provide limited information on the chemical state of surface cations and their distribution among facets, causing difficulty in explaining the corresponding facet-dependent results. Moreover, various processing steps and post-treatment techniques (e.g., $\mathrm{NaOH} /$ calcination treatment here) used during the preparation of NPs from group to group further complicate surface environments and always lead to different interpretations and disagreements among researchers. Herein, qualitative and quantitative information on both the chemical state and distribution of surface cations among facets promoted with various groups have been well resolved by the use of an NMR-active probe (Fig. 8). We are also able to monitor the electronic effect imposed by different adsorbates during sequential treatments/modifications to the NP surface cation (Supplementary Figs. 17 and 18). It is noted that the employment of a NMR chemical probe to aid the characterization of the acidity of zeolite was introduced firstly by Lunsford and colleagues $(1984)^{52}$ and is utilized for various solid acid catalysts nowadays ${ }^{53}$. However, to the best of our knowledge there is no report regarding its potential use in differentiating the adsorbate-dependent chemical state of cations on various crystal facets, which can be crucial for filling the gap between the model catalysts used in surface science and the real catalysts found in practical applications.

In conclusion, it is clear from this study that the type and quantity of surface Ti electronic states and structures are highly sensitive to the intrinsic nature and amount of particular facets, SDS and additives used as well as treatment methods adopted (calcination or $\mathrm{NaOH}$ wash), etc. They play decisive role(s) for a wide range of $\mathrm{TiO}_{2}$ applications, especially optical and thermal catalysis, which are closely associated with the coordination environment and the chemical state of surface titanium atoms at the catalyst surface. Hence, we believe that this study gives insights to resolve the current debates and confusions in some facet-dependent properties in literature, should more comprehensive surface characterization including ${ }^{31} \mathrm{P}$ adsorbate-assisted MAS NMR be invoked.

\section{Methods}

Synthesis of F-(001), F-(101) and powder $\mathrm{TiO}_{2}$ sample. A total of $5.0 \mathrm{~mL}$ of $\mathrm{Ti}\left(\mathrm{OC}_{4} \mathrm{H}_{9}\right)_{4}$ was mixed with a certain amount of hydrofluoric acid (40-48 wt.\%) in a Teflon-lined autoclave with a capacity of $40 \mathrm{~mL}$ and subsequently heated to $180^{\circ}$ $\mathrm{C}$ at a ramp rate of $2^{\circ} \mathrm{C} / \mathrm{min}$. The temperature was kept at $180^{\circ} \mathrm{C}$ for $24 \mathrm{~h}^{13}$. After hydrothermal reaction, the white precipitate was collected, washed with ethanol and distilled water three times and then dried in an oven at $80^{\circ} \mathrm{C}$ overnight. Then, 0.6 and $0.2 \mathrm{~mL}$ of hydrofluoric acid was added for F-(001) and F-(101), respectively, while, instead of hydrofluoric acid, $0.6 \mathrm{~mL}$ of $\mathrm{H}_{2} \mathrm{O}$ was employed for powder (PD) sample.

NaOH removal of surface fluorine. The $\mathrm{NaOH}$ wash was employed in this study according to previous literature ${ }^{13}$. Then, $1.0 \mathrm{~g}$ of as-prepared $\mathrm{TiO}_{2}$ sample was treated in $50.0 \mathrm{~mL}$ of $0.1 \mathrm{M} \mathrm{NaOH}$ solution under magnetic stirring for $10 \mathrm{~h}$ ( 0.05 and $0.5 \mathrm{M} \mathrm{NaOH}$ were also adopted for comparison). The solid sample was then washed with distilled water several times until neutral. After centrifugation, the solid was dried at $80^{\circ} \mathrm{C}$ overnight. $\mathrm{TiO}_{2}$ samples (i.e., PD, F-(101) and F-(001)) obtained after a $0.1 \mathrm{M} \mathrm{NaOH}$ wash were denoted as $\mathrm{Na}-\mathrm{PD}, \mathrm{Na}-(101)$ and Na-(001).

Calcination removal of surface fluorine. The calcination treatment was also carried out according to a previous report ${ }^{12}$. Then, $1.0 \mathrm{~g}$ of as-prepared $\mathrm{TiO}_{2}$ sample was calcined at $600{ }^{\circ} \mathrm{C}$ for $90 \mathrm{~min}$ (ramping rate: $5^{\circ} \mathrm{C} / \mathrm{min}$ ). We denoted $\mathrm{TiO}_{2}$ samples after receiving this treatment as Cal-PD, Cal-(101) and Cal-(001).

Sulfation of $\mathrm{TiO}_{\mathbf{2}}$. To obtain the sulfated $\mathrm{TiO}_{2}$, ammonium sulfate $\left(\left(\mathrm{NH}_{4}\right)_{2} \mathrm{SO}_{4}\right)$ was utilized as the precursor of sulfate in the catalyst preparation ${ }^{54}$. For example, $1.0 \mathrm{~g}$ of Na-PD/Cal-PD sample pretreated over a prolonged period of time was added into $10.0 \mathrm{~mL}$ of $1 \mathrm{~mol} / \mathrm{L}\left(\mathrm{NH}_{4}\right)_{2} \mathrm{SO}_{4}$ solution to stir for $8 \mathrm{~h}$ and then collected by centrifugation. The solid was then calcined at $450^{\circ} \mathrm{C}$ for $4 \mathrm{~h}$. We denoted $\mathrm{Na}-\mathrm{PD}$ and Cal-PD sample after sulfation as S-Na-PD and S-Cal-PD. The same treatments were also employed to the other two morphologies.

XPS, EPR and Raman measurement. XPS measurements were recorded on a Thermo Scientific K-Alfa XPS instrument equipped with micro-focused monochromated $\mathrm{Al} \mathrm{X}$-ray source. The source was operated at $12 \mathrm{keV}$ and a $400 \mu \mathrm{m}$ spot size was used. The analyzer operated at the analyzer energy (CAE) of $200 \mathrm{eV}$ for survey scans and $50 \mathrm{eV}$ for detailed scans. Charge neutralization was applied using a combined low energy/ion flood source. The data acquisition and analysis were conducted with CasaXPS (Casa software Ltd). The peak position was referenced to $\mathrm{C} 1 \mathrm{~s}$ peak of the carbon tape at $285.00 \mathrm{eV}$. The EPR spectra were obtained by X-band CW EPR spectrometer (Bruker EMX) and the signal intensity vs. electron spin number was obtained from the double integral of the spectrum. Raman spectra were measured with a Raman Microscope (Renishaw) with a laser excitation wavelength of $532 \mathrm{~nm}$. Exposure time of $10 \mathrm{~s}$ and 8 number scans were adopted for each measurement. 
TMP-adsorbed sample preparation. Approximately $150 \mathrm{mg}$ of $\mathrm{TiO}_{2}$ sample was placed in a home-made glass tube and activated at $150^{\circ} \mathrm{C}$ for $2 \mathrm{~h}$ under vacuum $\left(10^{-1} \mathrm{~Pa}\right)$ (Supplementary Fig. 19). After cool down to room temperature, $300 \mu \mathrm{mol} /$ catalyst $\mathrm{g}$ (calculated by the pressure and volume of isolated system) of TMP was then introduced. There was a wait of approximately $10 \mathrm{~min}$ for the equilibrium between TMP and catalyst surface to be reached. Extra TMP molecules were removed by vacuum system. These steps were repeated three times to ensure full adsorption of TMP on catalyst surface. The sample tube was then flame sealed for storage and transferred to Bruker $4 \mathrm{~mm} \mathrm{ZrO}_{2}$ rotor with a Kel-F endcap in a glove box under nitrogen atmosphere before NMR measurement (see Supplementary Note 6 for more details).

NMR measurement. The solid-state MAS NMR experiments were carried out using a Bruker Avance III 400WB spectrometer at room temperature. MAS speed of all our samples was $12 \mathrm{kHz}$. The high-power decoupling was used for the quantitative ${ }^{31} \mathrm{P}$ analysis. Considering the long relaxation time of ${ }^{31} \mathrm{P}$ nuclei in NMR experiment, we used $30^{\circ}$ pulse with the width of $1.20 \mu \mathrm{s}, 15 \mathrm{~s}$ delay time. The radiofrequency for decoupling was $59 \mathrm{kHz}$. The spectral width was 400 p.p.m., from 200 to -200 p.p.m. The number of scanning was 800 . The ${ }^{31} \mathrm{P}$ chemical shifts were reported relative to $85 \%$ aqueous solution of $\mathrm{H}_{3} \mathrm{PO}_{4}$, with $\mathrm{NH}_{4} \mathrm{H}_{2} \mathrm{PO}_{4}$ as a secondary standard (0.81 p.p.m.). The quantitative analysis of adsorbed TMP molecules was calculated according to the calibration line established by running standard samples with various adsorbed TMP concentration (see Supplementary Note 7, Supplementary Figs. 20-22 and Supplementary Table 7 for more details).

NMR spectrum deconvolution. All raw TMP NMR spectra were deconvoluted using the software 'peakfit v4.12'. We employed 'gauss area' and ensured all results with $R^{2}$ value of $>0.98$. Notice that the raw spectra data of samples with the same treatment/modification show s.d. of \pm 1 p.p.m. in chemical shift position. Supplementary Table 8 summarizes the positions fixed for the spectra deconvolution in Lewis acid region ( -20 to -58 p.p.m.). For example, during the spectral deconvolution of F-(101) and F-(001), we have fixed two positions of $\mathrm{Ti}_{5 \mathrm{c}}(101)$ and $\mathrm{Ti}_{5 \mathrm{c}}(001)$ at -31 and -22.5 p.p.m. within \pm 1 p.p.m. uncertainty. Also, for S-Na-PD, S-Na-(101) and S-Na-(001), the position of $\mathrm{Ti}_{5 \mathrm{c}}(101)$ and $\mathrm{Ti}_{5 \mathrm{c}}(001)$ were fixed at -25.5 and -34 p.p.m. within \pm 1 p.p.m. uncertainty.

Photocatalytic activity test. Comparative catalytic testing was conducted at room temperature, with a constant magnetic stirring to ensure full suspension of the particles. An aqueous $M G$ stock solution $(15 \mathrm{mg} / \mathrm{L})$ was used for all photoreactions. A total volume of $20 \mathrm{mg}$ of $\mathrm{TiO}_{2}$ sample was dispersed in $100 \mathrm{~mL}$ dye stock solution and stirred for $30 \mathrm{~min}$ in the dark to allow dye adsorption/desorption to equilibrate. The reaction mixture was then exposed to irradiation with constant stirring in a Luzchem photoreactor fitted with 8 ultraviolet A lamps with non-monochromatic irradiation centred at $350 \mathrm{~nm}$ resulting in a combined measured intensity of $4750 \mathrm{~lx}(\mathrm{Fc}=440)$.

Pechmann condensation catalytic activity test. The catalytic activities of $\mathrm{TiO}_{2}$ samples were studied for the synthesis of 5,7-dihydroxy-4-methyl coumarin from phloroglucinol and ethyl acetoacetate under solvent-free conditions. In a typical reaction, $5 \mathrm{mmol}$ of phloroglucinol was reacted with $10 \mathrm{mmol}$ of ethyl acetoacetate in the presence of $0.1 \mathrm{~g}$ of catalyst. The reaction mixture was kept at $130^{\circ} \mathrm{C}$ under reflux for a desired time. On completion of the reaction, the mixture was allowed to cool down to the room temperature followed by the addition of ethanol. The reaction mixture was filtered to separate the catalyst and the filtrate was analysed by liquid chromatography (DIONEX U3000). The yield of 5,7-dihydroxy-4-methyl coumarin was calculated as: Yield $(\%)=($ Obtained weight of product $) /($ Theoretical weight of product) $\times 100$.

DFT calculations. See Supplementary Note 8 and Supplementary Fig. 23 for the computational details of TMP adsorption energy and corresponding adsorption configuration on various $\mathrm{TiO}_{2}$ facets.

Data availability. The data that support this study are available from the corresponding author on request.

Received: 27 January 2017 Accepted: 13 July 2017

Published online: 22 September 2017

\section{References}

1. Boles, M. A., Ling, D., Hyeon, T. \& Talapin, D. V. The surface science of nanocrystals. Nat. Mater. 15, 141-153 (2016).

2. Zhang, X. et al. Effect of aspect ratio and surface defects on the photocatalytic activity of $\mathrm{ZnO}$ nanorods. Sci. Rep. 4, 4596 (2014).

3. Mueller, D. N., Machala, M. L., Bluhm, H. \& Chueh, W. C. Redox activity of surface oxygen anions in oxygen-deficient perovskite oxides during electrochemical reactions. Nat. Commun. 6, 6097 (2015).
4. Llordés, A. et al. Linear topology in amorphous metal oxide electrochromic networks obtained via low-temperature solution processing. Nat. Mater. 15 1267-1273 (2016).

5. Zandi, O. \& Hamann, T. W. Determination of photoelectrochemical water oxidation intermediates on haematite electrode surfaces using operando infrared spectroscopy. Nat. Chem. 8, 778-783 (2016).

6. Li, G. R. et al. Morphology-function relationship of ZnO: polar planes, oxygen vacancies, and activity. Phys. Chem. C 112, 11859-11864 (2008).

7. Peng, Y.-K. et al. Trimethylphosphine-assisted surface fingerprinting of metal oxide nanoparticle by ${ }^{31} \mathrm{P}$ solid-state NMR: a zinc oxide case study. J. Am. Chem. Soc. 138, 2225-2234 (2016).

8. Yin, Y. \& Alivisatos, A. P. Colloidal nanocrystal synthesis and the organic-inorganic interface. Nature. 437, 664-670 (2005).

9. Bakshi, M. S. How surfactants control crystal growth of nanomaterials. Cryst. Growth Des. 16, 1104-1133 (2016).

10. Liu, S., Yu, J. \& Jaroniec, M. Anatase $\mathrm{TiO}_{2}$ with dominant high-energy $\{001\}$ facets: synthesis, properties, and applications. Chem. Mater. 23, 4085-4093 (2011).

11. Liu, G. et al. Titanium dioxide crystals with tailored facets. Chem. Rev. 114, 9559-9612 (2014).

12. Yang, H. G. et al. Anatase $\mathrm{TiO}_{2}$ single crystals with a large percentage of reactive facets. Nature 453, 638-641 (2008).

13. Han, X., Kuang, Q., Jin, M., Xie, Z. \& Zheng, L. Synthesis of titania nanosheets with a high percentage of exposed (001) facets and related photocatalytic properties. J. Am. Chem. Soc. 131, 3152-3153 (2009).

14. Yang, H. G. et al. Solvothermal synthesis and photoreactivity of anatase $\mathrm{TiO}_{2}$ nanosheets with dominant $\{001\}$ facets. J. Am. Chem. Soc. 131, 4078-4083 (2009).

15. Liu, S., Yu, J. \& Jaroniec, M. Tunable photocatalytic selectivity of hollow $\mathrm{TiO}_{2}$ microspheres composed of anatase polyhedra with exposed $\{001\}$ facets. J. Am. Chem. Soc. 132, 11914-11916 (2010).

16. Pan, J., Liu, G., Lu, G. Q. \& Cheng, H.-M. On the true photoreactivity order of $\{001\},\{010\}$, and $\{101\}$ facets of anatase $\mathrm{TiO} 2$ crystals. Angew. Chem. Int. Ed. 50, 2133-2137 (2011).

17. Gordon, T. R. et al. Nonaqueous synthesis of $\mathrm{TiO}_{2}$ nanocrystals using $\mathrm{TiF}_{4}$ to engineer morphology, oxygen vacancy concentration, and photocatalytic activity. J. Am. Chem. Soc. 134, 6751-6761 (2012).

18. Yu, J., Qi, L. \& Jaroniec, M. Hydrogen production by photocatalytic water splitting over $\mathrm{Pt} / \mathrm{TiO}_{2}$ nanosheets with exposed (001) facets. J. Phys. Chem. C 114, 13118-13125 (2010).

19. Xiang, Q., Lv, K. \& Yua, J. Pivotal role of fluorine in enhanced photocatalytic activity of anatase $\mathrm{TiO}_{2}$ nanosheets with dominant $\left(\begin{array}{lll}0 & 0 & 1\end{array}\right)$ facets for the photocatalytic degradation of acetone in air. Appl. Catal. B 95, 557-564 (2010).

20. Jiang, Y., Scott, J. \& Amal, R. Exploring the relationship between surface structure and photocatalytic activity of flame-made $\mathrm{TiO}_{2}$-based catalysts. Appl. Catal. B 126, 290-297 (2012).

21. Tachikawa, T., Yamashita, S. \& Majima, T. Evidence for crystal-face-dependent $\mathrm{TiO}_{2}$ photocatalysis from single-molecule imaging and kinetic analysis. J. Am. Chem. Soc. 133, 7197-7204 (2011).

22. D'Arienzo, M. et al. Photogenerated defects in shape-controlled $\mathrm{TiO}_{2}$ anatase nanocrystals: a probe to evaluate the role of crystal facets in photocatalytic processes. J. Am. Chem. Soc. 133, 17652-17661 (2011).

23. Yu, J., Low, J., Xiao, W., Zhou, P. \& Jaroniec, M. Enhanced photocatalytic $\mathrm{CO}_{2}$-reduction activity of anatase $\mathrm{TiO} 2$ by coexposed $\{001\}$ and $\{101\}$ facets. J. Am. Chem. Soc. 136, 8839-8842 (2014).

24. Yu, X., Jeon, B. \& Kim, Y. K. Dominant influence of the surface on the photoactivity of shape-controlled anatase $\mathrm{TiO}_{2}$ nanocrystals. ACS Catal. $\mathbf{5}$, 3316-3322 (2015).

25. Xiong, F. et al. Methanol conversion into dimethyl ether on the anatase $\mathrm{TiO}_{2}$ (001) surface. Angew. Chem. Int. Ed. 55, 623-628 (2016).

26. Yu, J., Fan, J. \& Lv, K. Anatase $\mathrm{TiO}_{2}$ nanosheets with exposed (001) facets: improved photoelectric conversion efficiency in dye-sensitized solar cells. Nanoscale 2, 2144-2149 (2010).

27. Wu, X., Chen, Z., Lu, G. Q. \& Wang, L. Nanosized anatase $\mathrm{TiO}_{2}$ single crystals with tunable exposed (001) facets for enhanced energy conversion efficiency of dye-sensitized solar cells. Adv. Funct. Mater. 21, 4167-4172 (2011).

28. Hao, F. et al. Efficient light harvesting and charge collection of dye-sensitized solar cells with (001) faceted single crystalline anatase nanoparticles. J. Phys. Chem. C 116, 19164-19172 (2012).

29. Chu, L., Qin, Z., Yang, J. \& Li, X. Anatase $\mathrm{TiO}_{2}$ nanoparticles with exposed $\{001\}$ facets for efficient dye-sensitized solar cells. Sci. Rep. 5, 12143 (2015).

30. Chen, J. S. et al. Constructing hierarchical spheres from large ultrathin anatase $\mathrm{TiO}_{2}$ nanosheets with nearly $100 \%$ exposed (001) facets for fast reversible lithium storage. J. Am. Chem. Soc. 132, 6124-6130 (2010).

31. Cheng, X.-L., Hu, M., Huang, R. \& Jiang, J.-S. HF-free synthesis of anatase $\mathrm{TiO}_{2}$ nanosheets with largely exposed and clean $\{001\}$ facets and their enhanced rate 
performance as anodes of lithium-ion battery. ACS Appl. Mater. Interfaces 6, 19176-19183 (2014)

32. Selçuk, S. \& Selloni, A. Surface structure and reactivity of anatase $\mathrm{TiO}_{2}$ crystals with dominant $\{001\}$ facets. J. Phys. Chem. C 117, 6358-6362 (2013).

33. Yuan, W. et al. Real-time observation of reconstruction dynamics on $\mathrm{TiO}_{2}$ (001) surface under oxygen via an environmental transmission electron microscope. Nano. Lett. 16, 132-137 (2016).

34. Atanda, L., Mukundan, S., Shrotri, A., Ma, Q. \& Beltramini, J. Catalytic conversion of glucose to 5-hydroxymethyl-furfural with a phosphated $\mathrm{TiO}_{2}$ catalyst. ChemCatChem 7, 781-790 (2015).

35. Atanda, L. et al. Direct production of 5-hydroxymethylfurfural via catalytic conversion of simple and complex sugars over phosphated $\mathrm{TiO}_{2}$. ChemSusChem 8, 2907-2916 (2015).

36. Wang, X. C. et al. Probing of photocatalytic surface sites on $\mathrm{SO}_{4}{ }^{2-} / \mathrm{TiO}_{2}$ solid acids by in situ FT-IR spectroscopy and pyridine adsorption. J. Photochem. Photobiol. A 179, 339-347 (2006).

37. Zhang, $\mathrm{H}$. et al. Reactivity enhancement of 2-propanol photocatalysis on $\mathrm{SO}_{4}{ }^{2-} / \mathrm{TiO}_{2}$ : insights from solid-state NMR spectroscopy. Environ. Sci. Technol. 42, 5316-5321 (2008).

38. Hardin, B. E., Snaith, H. J. \& McGehee, M. D. The renaissance of dye-sensitized solar cells. Nat. Photonics 6, 162-169 (2012).

39. Kusama, H., Orita, H. \& Sugihara, $\mathrm{H}$. $\mathrm{TiO}_{2}$ band shift by nitrogen-containing heterocycles in dye-sensitized solar cells: a periodic density functional theory study. Langmuir 24, 4411-4419 (2008).

40. Lazzeri, M., Vittadini, A. \& Selloni, A. Structure and energetics of stoichiometric $\mathrm{TiO}_{2}$ anatase surfaces. Phys. Rev. B 63, 155409 (2001).

41. Chu, Y. et al. Acidic strengths of Brønsted and Lewis acid sites in solid acids scaled by ${ }^{31} \mathrm{P}$ NMR chemical shifts of adsorbed trimethylphosphine. J. Phys. Chem. C 115, 7660-7667 (2011).

42. Tedsree, K. ${ }^{13} \mathrm{C}$ NMR guides rational design of nanocatalysts via chemisorption evaluation in liquid phase. Science 332, 224-228 (2011).

43. Peng, Y.-K. et al. Probe-molecule-assisted NMR spectroscopy: a comparison with photoluminescence and electron paramagnetic resonance spectroscopy as a characterization tool in facet-specific photocatalysis. ChemCatChem $\mathbf{9}$, 155-160 (2017).

44. Ismagilov, Z. R. et al. Synthesis and stabilization of nano-sized titanium dioxide. Russ. Chem. Rev. 78, 873-885 (2009).

45. Belton, D. J., Deschaume, O. \& Perry, C. C. An overview of the fundamentals of the chemistry of silica with relevance to biosilicification and technological advances. FEBS J. 279, 1710-1720 (2012).

46. Yang, X. H., Li, Z., Sun, C., Yang, H. G. \& Li, C. Hydrothermal stability of $\{001\}$ faceted anatase $\mathrm{TiO}_{2}$. Chem. Mater. 23, 3486-3494 (2011).

47. Lv, K., Xiang, Q. \& Yu, J. Effect of calcination temperature on morphology and photocatalytic activity of anatase $\mathrm{TiO}_{2}$ nanosheets with exposed $\{001\}$ facets. Appl. Catal. B 104, 275-281 (2011).

48. Zhao, Y. et al. Shape-dependent acidity and photocatalytic activity of $\mathrm{Nb}_{2} \mathrm{O}_{5}$ nanocrystals with an active TT (001) Surface. Angew. Chem. Int. Ed. 51, 3846-3849 (2012).

49. Sudha, S. et al. Single step synthesis of coumarin derivatives over Al-MCM-41 and its supported catalysts under solvent-free condition. J. Mol. Catal. A Chem. 291, 22-29 (2008)

50. Yu, H., Fang, H., Zhang, H., Li, B. \& Deng, F. Acidity of sulfated tin oxide and sulfated zirconia: a view from solid-state NMR spectroscopy. Catal. Commun. 10, 920-924 (2009).
51. Yang, J. et al. Brönsted and Lewis acidity of the $\mathrm{BF}_{3} / \gamma-\mathrm{Al}_{2} \mathrm{O}_{3}$ alkylation catalyst as revealed by solid-state NMR spectroscopy and DFT quantum chemical calculations. J. Phys. Chem. B 109, 13124-13131 (2005).

52. Rothwell, W. P., Shen, W. \& Lunsford, J. H. Solid-state phosphorus-31 NMR of a chemisorbed phosphonium ion in HY zeolite: observation of protonphosphorus-31 coupling in the solid-state. J. Am. Chem. Soc. 106, 2452-2453 (1984).

53. Zheng, A., Huang, S.-J., Liu, S.-B. \& Deng, F. Acid properties of solid acid catalysts characterized by solid-state ${ }^{31} \mathrm{P}$ NMR of adsorbed phosphorous probe molecules. Phys. Chem. Chem. Phys. 13, 14889-14901 (2011).

54. $\mathrm{Hu}, \mathrm{Y}$. et al. Facet-dependent acidic and catalytic properties of sulfated titania solid superacids. Chem. Commun. 51, 14219-14222 (2015).

\section{Acknowledgements}

The financial support of this work from the EPSRC research council of UK is acknowledged. Y.-K.P. acknowledges a Clarendon Scholarship for his DPhil study at the Oxford University, UK. We thank Dr Gan-Zuei Gary Chang for the BET measurements.

\section{Author contributions}

Y.-K.P. carried out all relevant experiments, data analysis and wrote the first draft of the manuscript. Y.H. synthesized $\mathrm{TiO}_{2}$ samples and carried out catalytic experiments. All DFT calculations were performed by H.L.C. Y.F. and L.Z. prepared TMP-loaded sample and did MAS NMR measurement. I.F.T. did TEM for $\mathrm{TiO}_{2}$ samples. H.H. advised on NMR data and S.C.E.T. supervised this project.

\section{Additional information}

Supplementary Information accompanies this paper at doi:10.1038/s41467-017-00619-Z

Competing interests: The authors declare no competing financial interests.

Reprints and permission information is available online at http://npg.nature.com/ reprintsandpermissions/

Publisher's note: Springer Nature remains neutral with regard to jurisdictional claims in published maps and institutional affiliations.

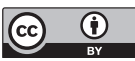

Open Access This article is licensed under a Creative Commons Attribution 4.0 International License, which permits use, sharing, adaptation, distribution and reproduction in any medium or format, as long as you give appropriate credit to the original author(s) and the source, provide a link to the Creative Commons license, and indicate if changes were made. The images or other third party material in this article are included in the article's Creative Commons license, unless indicated otherwise in a credit line to the material. If material is not included in the article's Creative Commons license and your intended use is not permitted by statutory regulation or exceeds the permitted use, you will need to obtain permission directly from the copyright holder. To view a copy of this license, visit http://creativecommons.org/ licenses/by/4.0/.

(c) The Author(s) 2017 